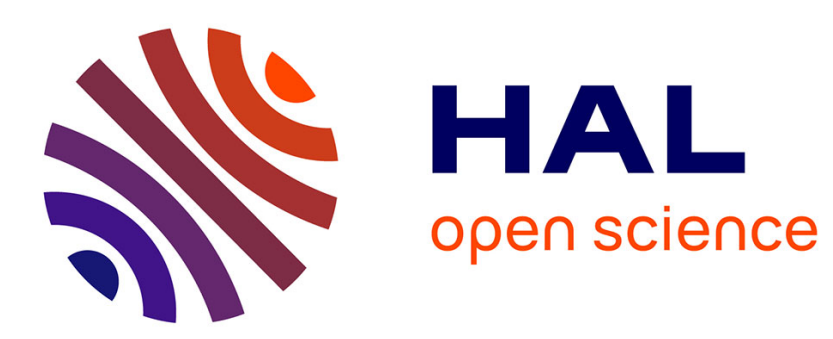

\title{
Instability mechanisms in swirling flows
}

François Gallaire, Jean-Marc Chomaz

\section{To cite this version:}

François Gallaire, Jean-Marc Chomaz. Instability mechanisms in swirling flows. Physics of Fluids, 2003, 15 (9), pp.2622-2639. 10.1063/1.1589011 . hal-01024935

\section{HAL Id: hal-01024935 \\ https://hal-polytechnique.archives-ouvertes.fr/hal-01024935}

Submitted on 27 Aug 2014

HAL is a multi-disciplinary open access archive for the deposit and dissemination of scientific research documents, whether they are published or not. The documents may come from teaching and research institutions in France or abroad, or from public or private research centers.
L'archive ouverte pluridisciplinaire HAL, est destinée au dépôt et à la diffusion de documents scientifiques de niveau recherche, publiés ou non, émanant des établissements d'enseignement et de recherche français ou étrangers, des laboratoires publics ou privés. 


\section{AIP $\mid$ Physics of Fluids}

\section{Instability mechanisms in swirling flows}

F. Gallaire and J.-M. Chomaz

Citation: Physics of Fluids (1994-present) 15, 2622 (2003); doi: 10.1063/1.1589011

View online: http://dx.doi.org/10.1063/1.1589011

View Table of Contents: http://scitation.aip.org/content/aip/journal/pof2/15/9?ver=pdfcov

Published by the AIP Publishing

\section{Articles you may be interested in}

Three-dimensional instability and state selection in an oscillatory axisymmetric swirling flow

Phys. Fluids 14, 3983 (2002); 10.1063/1.1509452

Three-dimensional swirling flow with a precessing vortex breakdown in a rotor-stator cylinder Phys. Fluids 13, 3500 (2001); 10.1063/1.1407268

The stability of noncolumnar swirling flows in diverging streamtubes

Phys. Fluids 13, 2835 (2001); 10.1063/1.1398043

Three-dimensional instability of anticyclonic swirling flow in rotating fluid: Laboratory experiments and related theoretical predictions

Phys. Fluids 10, 3194 (1998); 10.1063/1.869846

The effect of swirl on jets and wakes: Linear instability of the Rankine vortex with axial flow Phys. Fluids 10, 1120 (1998); 10.1063/1.869637

\section{A|P| Journal of}




\title{
Instability mechanisms in swirling flows
}

\author{
F. Gallaire and J.-M. Chomaz \\ Laboratoire d'Hydrodynamique (LadHyX), CNRS, Ecole Polytechnique, 91128 Palaiseau Cedex, France
}

(Received 14 March 2002; accepted 12 May 2003; published 5 August 2003)

\begin{abstract}
We investigate the stability of the screened Rankine vortex with added plug flow where the azimuthal velocity decreases abruptly outside the core of the vortex. The jump in circulation is known to induce centrifugal and azimuthal Kelvin-Helmholtz instabilities. Their effect on the stability of the different azimuthal wave number $m$ is discussed using physical considerations associated with asymptotic expansions and numerical computations of the dispersion relation. It is shown that the axial shear and centrifugal instability are active for all $m$, and that modes with $|m| \geqslant 2$ are also destabilized by azimuthal shear. In contrast, the bending modes $m= \pm 1$ are stabilized by a coupling with Kelvin waves in the core. Effects of rotation on the absolute/convective transition are also discussed. The absolute instability of positive helical modes is seen to be promoted by centrifugal instability and azimuthal shear. (c) 2003 American Institute of Physics.
\end{abstract}

[DOI: $10.1063 / 1.1589011]$

\section{INTRODUCTION}

Coherent structures and their stability are known to be very important in geophysical ${ }^{1}$ or industrial flows. ${ }^{2}$ They control momentum or passive scalar (often pollutants) transfer from one scale to another and from one physical location to another. For large-scale geophysical flows these structures are mainly two-dimensional vortices, famous examples being the earth polar vortex and the Jovian red spot. Many studies have been devoted to the stability of two-dimensional (2-D) screened vortices since the instability that develops at the periphery of such a structure will act as a precursor to mixing between the vortex core and the surrounding fluid that will be crucial, e.g., in the depletion of the Earth's ozone. ${ }^{3}$ In that 2-D case, centrifugal effects are not active and the dynamics is only governed by the azimuthal shear. In particular, Carton and McWilliams ${ }^{4}$ and Carnevale and Kloosterziel ${ }^{5}$ have shown that, due to the azimuthal shear, instabilities of wave numbers $m=2,3,4$ develop in the region of azimuthal shear, eventually leading to stable dipoles, tripoles, or quadrupoles. Similar results have recently been obtained by the numerical simulation of Bergeron et al. ${ }^{6}$ Experimentally, the dynamics of 2-D screened vortices have been studied either in a closed geometry ${ }^{7-9}$ or in an open parabolic tank, ${ }^{10}$ and the circular shear layer was observed to roll up into a finite, eventually large number of barotropic vortices, that subsequently pair due to a secondary instability. In all the experiments the number of vortices that appeared was roughly proportional to the ratio of the circular shear layer diameter to its thickness.

In the planetary atmosphere or in the ocean, the 2-D approximation (or equivalently the barotropic approximation) breaks down when vortices are of small scale or when equatorial flows are considered. In this case the mean rotation ceases to dominate and three-dimensional (3-D) instabilities may occur. Since the azimuthal shear is also associated with a decrease in azimuthal circulation, centrifugal instabilities are likely to render the flow three-dimensional similar to Taylor-Couette experiments. This centrifugal in- stability has been used to explain the asymmetry between cyclonic and anticylonic motion observed in laboratory experiments, ${ }^{11}$ Jovian atmosphere, ${ }^{12}$ or on Earth ${ }^{1}$ when the local Rossby number quantifying the local vorticity compared to the mean rotation is larger than unity. Experimentally, the centrifugal instability has been observed by Afanasyev and Peltier ${ }^{13}$ and by Colette et al.,${ }^{14}$ who report that the destabilization occurs through an axisymmetric mode $(m=0)$ as in the Taylor-Couette or Dean ${ }^{15}$ experiment. However, in the presence of axial flow, the bulging mode $m=0$ is not the only mode destabilized by the decrease in circulation, as shown theoretically by Ludwieg ${ }^{16}$ and Leibovich and Stewartson, ${ }^{17}$ who have extended the Rayleigh criterion for centrifugal axisymmetric instabilities to nonaxisymmetric perturbations. In the case $m \neq 0$, both azimuthal Kelvin-Helmholtz instabilities and centrifugal instabilities should be actually taken into account, even in the presence of axial flow. The destabilization of a tornado into a multiple vortex mode with three or four tornadoes described by Snow ${ }^{18}$ is due to such a spiral instability mode when the tornado is viewed as a screened swirling jet. In industrial applications, swirling flows are commonly used in burners as flame stabilization devices or in the laboratory as models for the study of vortex breakdown. ${ }^{19}$ In that case, the swirling jet produced at the nozzle directly enters a large quiet tank. Away from the jet axis the circulation decreases rapidly to zero. In other experiments such as the one by Escudier et al. ${ }^{20}$ the swirling flow is confined in a diverging tube, and the boundary layer is also subject to centrifugal (Görtler) and shear instabilities.

The temporal as well as spatiotemporal stability of swirling jets and wakes has received considerable attention in recent years. The temporal studies of the well-known Batchelor vorte ${ }^{21-23}$ have recently been completed by spatiotemporal studies ${ }^{24-27}$ to analyze in detail the behavior of "smooth" swirling jets that are stable with respect to axisymmetric centrifugal and Kelvin-Helmholtz instabilities. 
Simple swirling jet models, for which the dispersion relation can be derived analytically, have been also introduced, to better understand the competition between azimuthal and streamwise vorticity. In their study of the Rankine vortex (consisting of a core in solid body rotation surrounded by a potential flow with constant circulation) with top-hat axial velocity profile, Loiseleux et al. ${ }^{28}$ (further referred to as LCH) have pointed out the stabilizing role of the swirl through a coupling between the Kelvin waves and the axial shear Kelvin-Helmholtz mode. These authors have also shown that the absolute nature of instabilities was enhanced by swirl, both for swirling jets and wakes. The Caflish vortex with top-hat axial velocity profile also received a lot of attention. ${ }^{29-31}$ Its stability properties were seen to depend on whether the vortex was centrifugally stable or unstable. Its core is modeled by a filament and cannot sustain Kelvin waves. Finally, Lim and Redekopp ${ }^{31}$ introduced a screened Rankine vortex with a top-hat axial velocity profile, which is intermediate between the two previous models, since the circulation decreases at the vortex periphery like in the centrifugally unstable Caflish model and, on the other hand, the inner core is in solid body rotation typical of a Rankine vortex. In the spirit of Monkewitz and Sohn, ${ }^{32}$ Lim and Redekopp ${ }^{31}$ have investigated variable density ratio effects, which are known to have a strong influence on the $\mathrm{A} / \mathrm{C}$ nature of the instabilities. But they have merely determined the absolute/ convective transition for the axisymmetric mode $m=0$. For a homogeneous swirling jet in a medium at rest, the axisymmetric $m=0$ mode is seen to remain convective when the swirl is increased for swirl parameters studied by the authors.

It is worth reexamining this rich swirling jet model in the case of three-dimensional perturbations with $m$ nonequal to zero, because inertial effects in the rigidly rotating core, centrifugal force, axial and azimuthal shear are then all in interplay, as they most generally are in experimental profiles. In the present study we first establish the temporal instability curves, analyzing thereby the role of the different mechanisms by means of asymptotic expansions and physical considerations. The A/C transition curves are then determined for all azimuthal wave numbers $m$ both for jets and wakes. For large enough swirl parameters, the predominance of positive helical modes for wakes is clearly established, as well as for jets. Only for a small swirl parameter does the axisymmetric mode $m=0$ become first absolutely unstable. For higher swirl numbers, large azimuthal wave numbers first become absolutely unstable, though for high swirl jets this result should be considered with care because the same difficulties in the application of the pinching point criterion as those mentioned by Lim and Redekopp ${ }^{31}$ or LCH are encountered. Finally, though the present model faithfully describes swirling jet experiments for which the circulation drops to zero outside the core, it lacks an essential ingredient to make it fully realistic: the shear is infinitely concentrated in the model whereas in experiments the shear layer thickness is finite. For the planar mixing layer (Drazin and Reid $^{15}$ ), the finite thickness is known to modify radically the temporal stability properties at high wave number. Keeping in mind that the effect of a finite shear thickness may only be addressed rigorously by a full stability analysis of real pro-

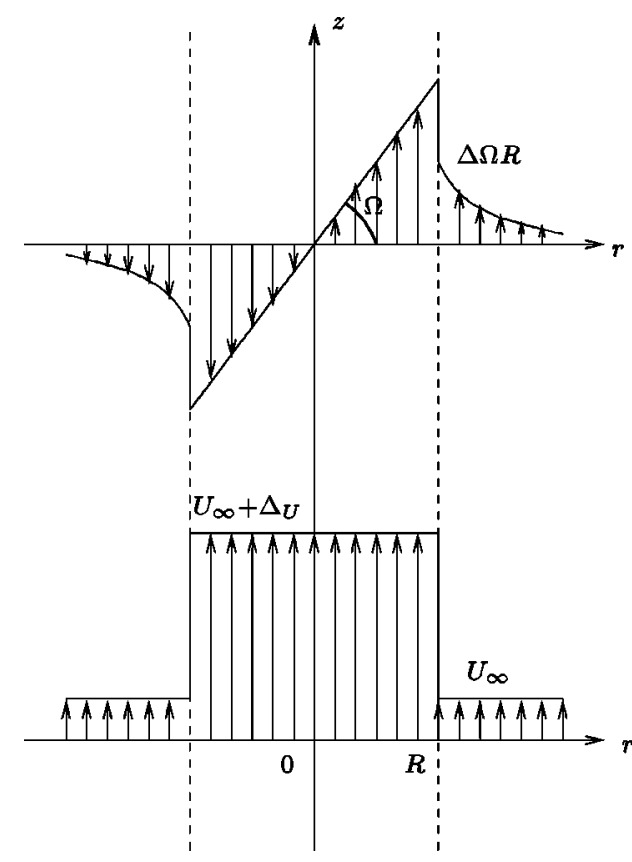

FIG. 1. Screened Rankine vortex with plug flow.

files (a study beyond the scope of the present paper), we tentatively discuss the robustness of the present model results based on physical arguments.

Our paper is organized as follows. In the first section, the base flow is defined and some physical considerations are presented, letting the derivation of the dispersion relation for Sec. III. Section IV is devoted to asymptotic expansions and numerical computations. In addition to the results of the first section, the dominant physical mechanisms are thereby identified. In Sec. V we discuss the absolute/convective (A/C) nature of the instability under the influence of the mean advection and magnitude of rotation. Finally, some conclusions and discussions are drawn in the last section.

\section{BASIC FLOW}

\section{A. Screened Rankine vortex with plug flow}

The basic swirling flow under consideration is a screened Rankine vortex with plug flow, as introduced by Lim and Redekopp. ${ }^{31}$ We assume the fluid to be inviscid. The basic flow sketched in Fig. 1 is defined as the following:

$$
\begin{aligned}
& \text { If } r \leqslant R: \quad U_{r}=0 ; \quad U_{\theta}=\Omega r ; \quad U_{z}=U_{j}=U_{\infty}+\Delta_{U} ; \\
& P=P_{\infty}-\rho \frac{\Omega^{2} \Delta^{2} R^{2}}{2}+\rho \frac{\Omega^{2}}{2}\left(r^{2}-R^{2}\right) ; \\
& \text { if } r>R: \quad U_{r}=0 ; \quad U_{\theta}=\frac{\Omega \Delta R^{2}}{r} ; U_{z}=U_{\infty} ; \\
& P=P_{\infty}-\rho \frac{\Omega^{2} \Delta^{2} R^{4}}{2 r^{2}},
\end{aligned}
$$

and represents a solution of the Euler equations. The main characteristics of the basic flow are easily identified: an axial flow with the shear $\Delta_{U}$ at radius $R$, a core of radius $R$ in solid body rotation with angular velocity $\Omega$ surrounded by a po- 
tential flow extending to $r \rightarrow \infty$ with a circulation $\Gamma$ $=\Omega R^{2} 2 \pi \Delta$ (where $\Delta \in[0,1]$ ). When $\Delta=0$ the vortex is referred to as totally screened and an azimuthal velocity shear occurs at the radius $R$ from $\Omega R$ to 0 . When $\Delta$ is nonzero, the vortex is only partially screened and when $\Delta=1$ the azimuthal velocity is continuous and the usual Rankine vortex is recovered as in LCH. We will focus on $\Delta=0$, which applies to free swirling jets, however, varying $\Delta$ will allow us to identify more easily the contribution of different instability mechanisms. In order to describe this family of vortices, we nondimensionalize using $R$ and $\Delta_{U}$ as length and velocity scales, respectively. This leads to the following nondimensional parameters that characterize the flow.

$a=U_{\infty} / \Delta_{U}$, the ratio of the outer axial stream to axial shear. The external and core flows are coflowing when $a \leqslant-1$ or $a \geqslant 0$ and counterflowing when -1 $\leqslant a \leqslant 0$. Jets correspond to $a \geqslant-0.5$ and wakes to $a$ $\leqslant-0.5$. Of course, a change in $a$ is equivalent to a change of Galilean reference frame. Therefore, it does not affect the temporal instability theory and is relevant only for the spatial instability results and for the determination of absolute/convective instabilities.

(ii) $S=\Omega R / \Delta_{U}$, the swirl (as defined in LCH) that expresses the importance of the rotation of the core versus the axial shear.

(iii) $\Delta$, the ratio of outer to inner circulation. The product $(1-\Delta) S$ is a measure of the azimuthal shear with respect to the axial shear.

This model combines the instability mechanisms of a jet $^{33}$ and a screened vortex column as discussed in the Introduction and its dynamics involves four different physical mechanisms.

(i) Axial shear gives rise to axial Kelvin-Helmholtz instabilities well known in nonrotating jets. ${ }^{33}$

(ii) The solid rotation of the core supports confined inertial waves, as we will discuss in the next section. Physically, inertial waves are due to the restoring action of the Coriolis force in the reference frame rotating with the vortex core. For the Rankine vortex without axial flow, the core acts as a wave guide and the resulting discrete set of inertial waves corresponds to Kelvin waves as described by Saffman. ${ }^{34}$

(iii) Centrifugal instabilities are active when $\Delta<1$ : the circulation decreases between the core and the surrounding fluid and Rayleigh's criterion implies that axisymmetric perturbations are centrifugally unstable.

(iv) Azimuthal shear destabilizes the flow when $\Delta<1$ or $\Delta>1$ since the associated jump in azimuthal velocity induces an azimuthal Kelvin-Helmholtz instability fully described in the 2-D context, i.e., in the absence of axial flow, by Carton and McWilliams ${ }^{4}$ and Carnevale and Kloosterziel. ${ }^{5}$

It is worthwhile distinguishing between centrifugal and azimuthal shear instabilities. We will explain in the next section that, while the latter originates in a difference of azimuthal speed, centrifugal instabilities result from a stratifica- tion in kinetic momentum. Furthermore, centrifugal instabilities are three dimensional in the sense that they do not exist for a 2-D flow, whereas the azimuthal KelvinHelmholtz instability does. They both also correspond to two different criteria, both attributed to Rayleigh. On the one hand, the well-known Rayleigh criterion for centrifugal axisymmetric $(m=0)$ instability stipulates that an instability occurs if the square of the angular momentum $r^{2} U_{\theta}^{2}$ decreases with $r$. This sufficient condition reads as

$$
U_{\theta}(r) \times W(r)<0,
$$

at some $r$, where $W(r)=(1 / r)\left(\partial r U_{\theta} / \partial r\right)$ is the axial vorticity. On the other hand, a necessary condition for purely 2-D shear instability $(k=0)$, also due to Rayleigh, is the extension to circular geometry of the inflection point theorem and requires a change in the sign of $d W(r) / d r .{ }^{15}$ Therefore, if the circulation of a vortex decreases, as in the present model for $\Delta<1$, both instability mechanisms are active whereas if the circulation increases $(\Delta>1)$, only the azimuthal shear instability is active, which is the case for a core with a faster annular flow outside.

\section{B. The physics of the instabilities}

Before deriving the dispersion relation in the next section, we study separately each physical mechanism in order to gain some physical insight in the dynamics involved. We first consider the neutral Kelvin modes sustained by the solid rotation of the core and show that they induce a cut-off frequency for the penetration of perturbations inside the core. The role of shear and centrifugal instability are then analyzed by estimating the associated destabilizing forces as a function of the axial wave number $k$ and azimuthal wave number $m$.

\section{Solid rotation of the core and role of the Coriolis force}

We will briefly recall some well-known ${ }^{34}$ results about Kelvin waves and their interpretation in terms of inertial waves for which the vortex core plays the role of a wave guide.

In a rotating media of infinite extension, the Coriolis force may be viewed as a restoring force that opposes the displacement of particles normal to the rotation axis. It leads to inertial waves, associated to the dispersion relation $\omega_{j}$ $=2 \Omega \cos (\theta)$, where $\theta$ is the angle between the rotation axis and the wave vector and where $\omega_{j}$ is the inertial wave frequency in the rotating frame. In the rotating frame, their frequency is therefore limited by a cut-off at $\pm 2 \Omega$ and, consequently, their frequency in the laboratory frame belongs to ] $m \Omega-2 \Omega, m \Omega+2 \Omega[$.

The introduction of the confinement of the waves in a cylindrical core in solid body rotation discretizes this continuous set of inertial waves. Building on the work of Kelvin ${ }^{35}$ and studying the unbounded Rankine vortex (without axial flow and $\Delta=1$ ), Saffman ${ }^{34}$ demonstrates for each azimuthal wave number $m$ the existence of an infinity of Kelvin modes with a frequency given in the low-wave number limit $(k R \ll 1)$ by 
(a)

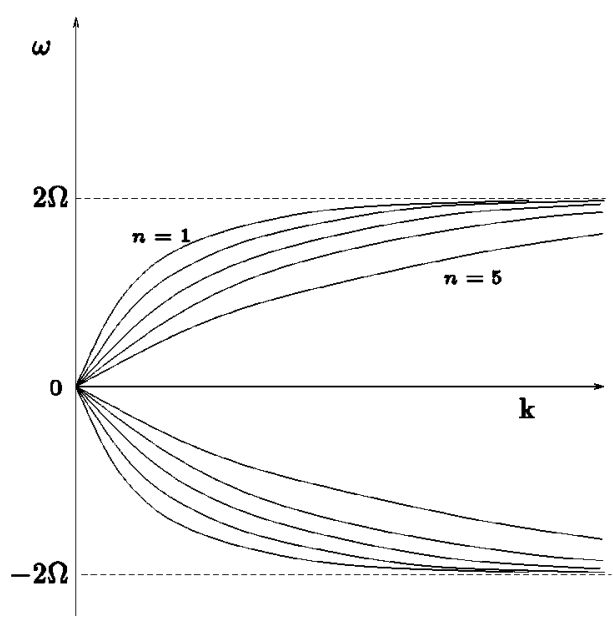

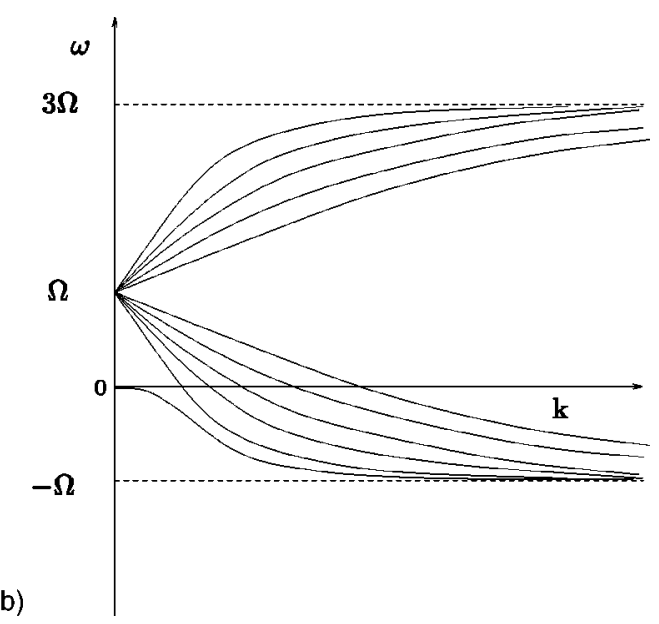

FIG. 2. Kelvin waves of the Rankine vortex with $\Delta=1$ and $\Delta_{U}=0$ [following Saffman (Ref. 34)]; (a) $m=0$, (b) $m=+1$.

$$
\omega=m \Omega \pm \frac{2 \Omega}{j_{m, n}} k R,
$$

where $j_{m, n}$ is the $n$th zero of the Bessel function $J_{m}$.

All these modes have a frequency equal to $m \Omega$ at $k$ $=0$ and a finite group velocity of $\pm 2 \Omega / j_{m, n}$. The frequencies of the modes as a function of $k$ are shown for the bulging mode $m=0$ and one bending mode $m=1$ in Fig. 2. They separate into cograde and retrograde ${ }^{17}$ modes according to the sign of the group velocity at $k=0$.

Note the existence of a particular mode with vanishing group velocity at $k=0$ in Fig. 2(b). This special mode that exists for $m \neq 0$ and has, at $k=0$, a frequency equal to $\Omega(m-\operatorname{sign}(m))$ as well as a vanishing group-velocity; it is called a slow mode. ${ }^{34}$

We shall retain that for each $m$, there exists a countable infinity of neutral modes of frequency belonging to the passing band ] $m \Omega-2 \Omega ; m \Omega+2 \Omega$ [ that corresponds to the passing band of unconfined inertial waves.

\section{Kelvin-Helmholtz instability}

In our swirling jet model, the shear layer is formed by the interface between the core in solid body rotation and the external potential flow. We assume, in a first approximation, that the perturbed flow is potential on both sides of the shear layer. More precisely, the deformation at the interface combines an axial perturbation (of wave number $k$ ) and an azimuthal deformation of wave number $m$. If $m$ and $k R$ are large enough, the curvature effect on the shear is expected to be negligible. The shear at the border of the jet can therefore be interpreted as a plane 2-D velocity jump, $\Delta_{U}$, in the $\left(e_{z}\right)$ direction and $S(1-\Delta) \Delta_{U}$ in the azimuthal direction $\left(e_{\theta}\right)$. This plane shear, associated with the total shear vector $\Delta \vec{U}_{\text {tot }}=\Delta_{U}\left[\vec{e}_{z}+S(1-\Delta) \vec{e}_{\theta}\right]$, is then perturbed by a 2-D wave vector $\vec{k}_{\text {tot }}=k \vec{e}_{z}+(m / R) \vec{e}_{\theta}$. The growth rate (cf. Drazin and Reid $^{15}$ ) reads as

$$
\sigma=\frac{1}{2}\left|\vec{k}_{\text {tot }} \cdot \Delta \vec{U}_{\text {tot }}\right|=\frac{1}{2}\left|\Delta_{U}\left(k+\frac{m S}{R}(1-\Delta)\right)\right| .
$$

In nondimensional variables, this leads to the expression

$$
\sigma=\frac{1}{2}|k+m S(1-\Delta)|,
$$

which will be later referred to as the "tilting shear" approximation. For a given $k \geqslant 0$, Eq. (5) shows that the more positive $m$, the stronger the instability. Each negative $-m$ mode is less unstable than its positive $+m$ counterpart since the total wave vector $\vec{k}_{\text {tot }}$ is "less" aligned with the total shear $\Delta \vec{U}_{\text {tot }}$.

Estimate (5) takes into account neither the centrifugal instability nor the effect of the core in solid body rotation. To understand how these effects may interact with the KelvinHelmholtz instability, we first recall the physical mechanism that drives the shear instability.

As before, let us first assume that the perturbed flow is potential on both sides of the shear layer. We take place in the reference frame moving at the mean speed of the two layers and consider an artificially frozen stationary interface deformation of wavelength $\lambda$ and amplitude $\mathcal{A}$. This deformation modifies the streamline pattern away from the interface on a length scale equal to $\lambda$, as shown in Fig. 3 (see the discussion below). Convergence of the streamlines implies acceleration of the fluid and, therefore, according to Bernoulli's theorem, a pressure drop. Conversely, divergence of the streamlines causes a deceleration of the fluid and hence an increase in pressure. The perturbation pressure gradient across the interface that would exist if the interface were frozen is balanced by the acceleration of the fluid normal to the interface and causes infinitesimal perturbations to grow. This destabilizing effect of the dynamical pressure is at the base of the Kelvin-Helmholtz instability.

As outlined previously, in order to compare the KelvinHelmholtz shear mechanism to other physical effects present in the swirling jet, the destabilizing force is estimated. We freeze the interface and use the steady version of Bernoulli's theorem along a streamline to estimate the pressure variation between the unperturbed point A where the velocity is $\bar{U}$ and point $\mathrm{B}$, located at a distance $\lambda / 4$ from $\mathrm{A}$, where the velocity is $\bar{U}+\delta U$,

$$
\delta p=\rho \bar{U} \delta U
$$




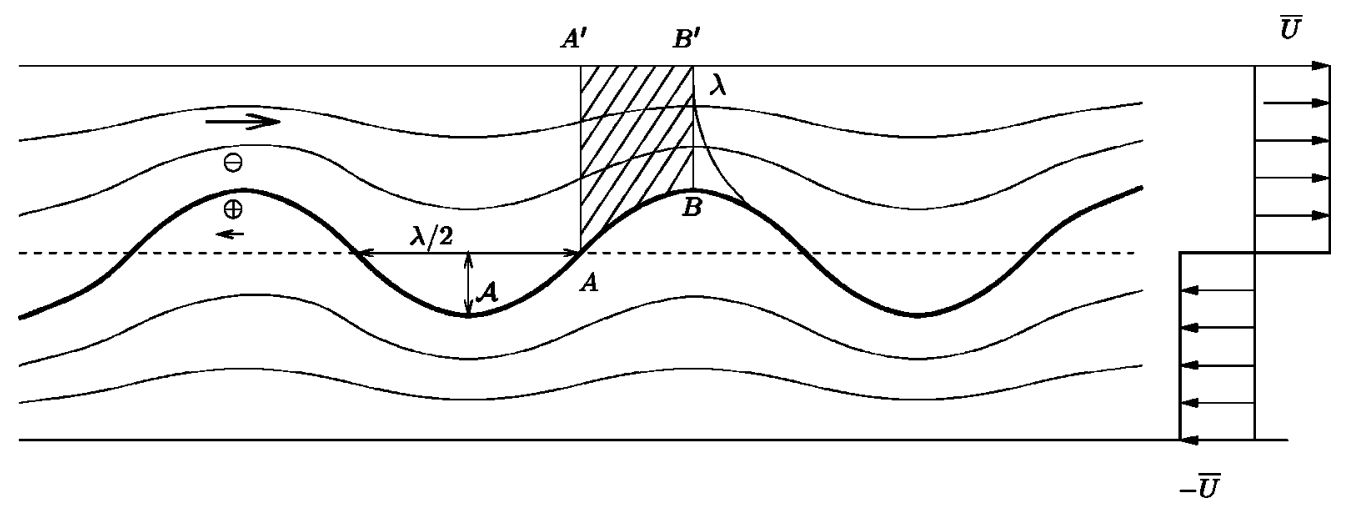

FIG. 3. Kelvin-Helmholtz shear layer instability mechanism.

The scaling of the velocity perturbation $\delta U$ with respect to the amplitude of the wave, $\mathcal{A}$, crucially depends on the distance along which the flow lines are bent. The irrotational nature of the flow outside of the interface causes a sinusoidal deformation in $\sin (k z)$ to generate a flow perturbation that vanishes away from the interface like $e^{-k y}$. In other words, the velocity perturbations decay away from the interface on a length scale of order $\lambda$ and the conservation of the flow-rate in the flow-tube $\mathrm{AA}^{\prime} \mathrm{BB}^{\prime}$ (cf. Fig. 3) gives

$$
\delta U \sim \frac{\mathcal{A} \bar{U}}{\lambda} .
$$

The variation of pressure therefore equals

$$
\delta p \sim \frac{\rho \bar{U}^{2} \mathcal{A}}{\lambda} .
$$

The destabilizing force per square unit length is hence proportional to the wave number $k$,

$$
F_{\mathrm{KH}} \sim \rho \frac{\bar{U}^{2} \mathcal{A}}{\lambda} \sim \rho \bar{U}^{2} k \mathcal{A} .
$$

What are the points hidden behind this argument, which fail when the 2-D plane potential shear layer assumption is relaxed? First, we have taken advantage of the $y \leftrightarrow-y$ symmetry of the base flow and assumed implicitly that the instability preserves this symmetry, implying, in particular, that its phase velocity is zero in that frame. In another frame of reference, where the phase velocity $v_{\phi}$ is nonzero, the unsteady Bernoulli theorem should have been used to estimate the driving force of the instability. This results in

$$
\delta p \sim \frac{\rho \mathcal{A}}{2 \lambda}\left(\left|U_{1}-v_{\phi}\right|^{2}+\left|U_{2}-v_{\phi}\right|^{2}\right),
$$

with $U_{1}$ (resp., $U_{2}$ ) as the speed in the upper layer (resp., lower layer). From the symmetry argument invoked above we know that $v_{\phi}=\left(U_{1}+U_{2}\right) / 2$ for the Kelvin-Helmholtz instability. If another instability mechanism modifies the phase speed, formula (10) describes the change in the destabilizing force. Of course, if the symmetry is broken (as it is for circular jets), the phase velocity is given by the complete solution of the dispersion relation [see Eq. (21) in the next section] and the present physical analysis determines how the Kelvin-Helmholtz instability is modified.

The second subtle point concerns the knowledge of the crosswise penetration of the perturbation in order to estimate the velocity defect that generates the destabilizing pressure difference. For Kelvin-Helmholtz instability, the decrease is exponential since the flow is potential outside the shear layer. But if vorticity is present on one side of the interface, the flow is no longer potential, and the penetration length and therefore the destabilizing pressure are modified. This is the case for swirling jets since vorticity is present inside the core. Indeed, when $\omega_{j}$, the frequency of the perturbation in

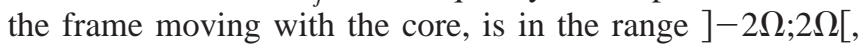
inertial waves (Kelvin waves) may be excited inside the vortex. In that case the penetration length on the core side of the shear becomes infinite (in fact, limited by the radius $R$ ), the velocity perturbations parallel to the interface vanish on that side and so does the pressure that was previously driving the instability (see Fig. 4). This demonstrates how the coupling with inertial waves may be stabilizing and elucidates former ${ }^{28}$ results on the Rankine vortex with no azimuthal shear. $\mathrm{LCH}^{28}$ have effectively showed that there is a resonance between the Kelvin waves contained in the core and the axial Kelvin-Helmholtz $m=1$ instability wave, leading to a stabilization. The physical argument proposed here limits the possibility of such a stabilizing coupling to cases where the frequency $\omega_{j}$ in the core is in the range ] $-2 \Omega ; 2 \Omega$ [. This will be confirmed in the present analysis.

\section{Centrifugal instability}

To estimate centrifugal effects, one considers, as before, perturbations with $k R$ and $m$ sufficiently large. In the core, the centrifugal force equals $U_{\theta}^{2}(r) / r=\Omega^{2} r$. Close to the interface but still inside the core, it is compensated by the centrifugal pressure gradient that maintains equilibrium $\partial_{r} P\left(R^{-}\right)=\rho U_{\theta}^{2}\left(R^{-}\right) / R$ where the notation $R^{-}$(resp., $R^{+}$) means that, for any function $f(r), f\left(R^{-}\right)$[resp., $f\left(R^{+}\right)$] is the value of $f$ as $r$ tends to $R$ from below (resp., from above). Outside the core the azimuthal velocity is, in general, not zero and also there a pressure gradient counteracts the centrifugal force. Its variation in $r$, however, differs from the expression inside the core. The pressure gradient close to the 


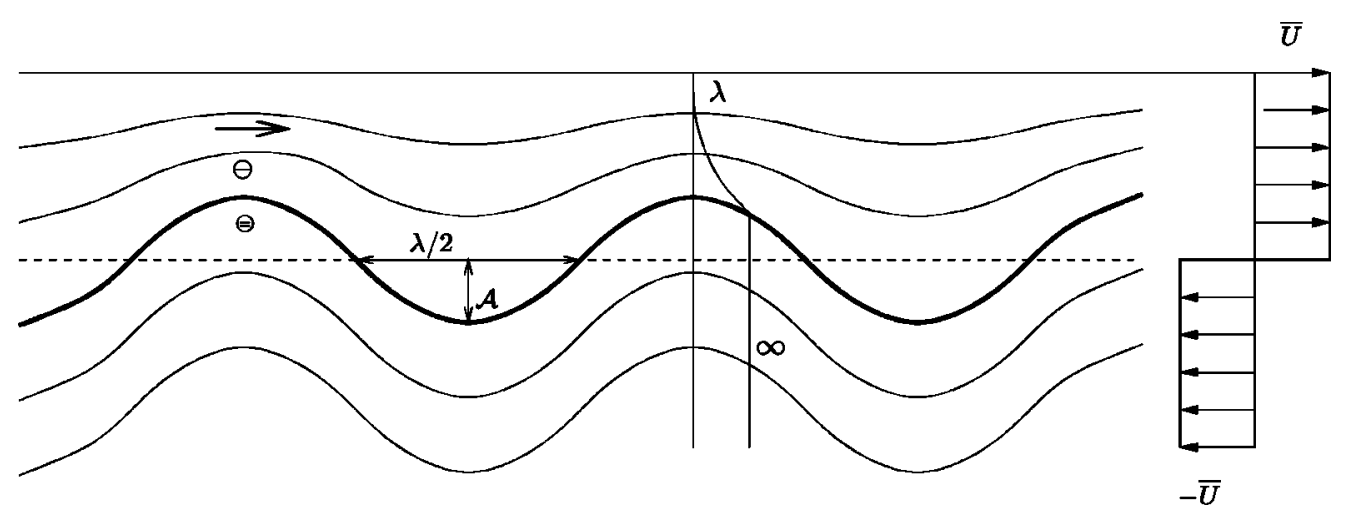

FIG. 4. The rotating core reduces the strength of the $\mathrm{K}-\mathrm{H}$ instability.

interface but outside the core equals $\partial_{r} P\left(R^{+}\right)$ $=\rho U_{\theta}^{2}\left(R^{+}\right) / R$. Centrifugal instability occurs when $\partial_{r} P\left(R^{-}\right)$ is larger than $\partial_{r} P\left(R^{+}\right)$.

In the present case, as soon as $\Delta \neq 1$, due to of the azimuthal velocity jump, the centrifugal force is lower outside the core than inside and is balanced by a smaller pressure gradient (see Fig. 5). When the interface undergoes a deformation, a fluid particle from the core displaced outside keeps experiencing a high centrifugal force when we assume that it conserves its angular momentum (the fluid being inviscid) while it is subjected to a smaller pressure gradient. The balance is reversed when a particle from outside is displaced toward the core since now it experiences a large pressure gradient and a small centrifugal force. The equilibrium between centrifugal forces and radial pressure is therefore unstable and any initial deformation will be amplified by this mechanism. An estimate for the force per unit square length is

$$
F_{c}=\rho \Omega^{2}\left(1-\Delta^{2}\right) \mathcal{A} R,
$$

and does not depend on $\lambda$ contrary to the $\mathrm{K}-\mathrm{H}$ instability force that is inversely proportional to $\lambda$. Comparing Eq. (9) and Eq. (11), we expect a dominance of the shear over the centrifugal effect when $k_{\text {tot }}$ is large.

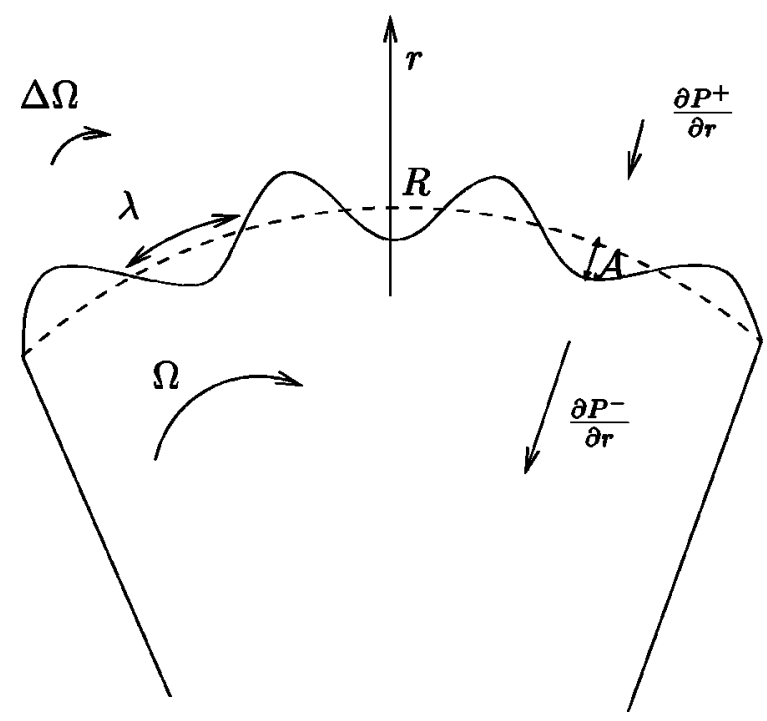

FIG. 5. Centrifugal destabilizing force.

\section{THE DISPERSION RELATION}

Although the computation is rather standard, ${ }^{31}$ it is detailed here to emphasize the physics embedded in the mathematical expression. The Euler equations are linearized around the base flow and normal mode perturbations in the axial and azimuthal directions are assumed. Since the basic flow is discontinuous at $r=R$, perturbations in velocity and pressure separate into two expressions valid in the core of the vortex and outside the core of the vortex, and are linked by jump conditions across the interface $r=R$.

a. Core structure. In the core region, the basic flow is a solid-body rotation and the perturbed solution takes the form

$$
\begin{aligned}
& u_{r}(r)=i A_{1} \frac{k^{2}}{\rho \omega_{j} \beta^{2}}\left(\frac{-2 m \Omega}{r \omega_{j}} J_{m}(\beta r)+\beta J_{m}^{\prime}(\beta r)\right), \\
& p(r)=A_{1} J_{m}(\beta r),
\end{aligned}
$$

where $u$ and $p$ are the perturbed velocity and pressure, $J_{m}$ is the modified Bessel function of order $m, A_{1}$ is a constant, and $\omega_{j}$ is the frequency in the frame rotating and translating with the core fluid,

$$
\omega_{j}=\omega-m \Omega-U_{j} k,
$$

$\beta$ is given by

$$
\beta^{2}=k^{2}\left(\frac{4 \Omega^{2}}{\omega_{j}^{2}}-1\right) .
$$

When $\beta^{2}$ is positive, this perturbation corresponds to a trapped internal wave associated with the Coriolis force, as discussed in Sec. II B 1. The cut-off effect on the frequency $\omega_{j}$ is recovered, considering that, for $\omega_{j}$ real, if $\omega_{j}<2 \Omega, \beta$ is real, the Bessel function $J_{m}$ is oscillatory in structure, and the eigenfunction is of order unity everywhere inside the core whereas if $\omega_{j}>2 \Omega, \beta$ is purely imaginary and the perturbation is evanescent from the interface to the axis.

$b$. Outer structure. The solution in the outer region corresponds to a potential flow perturbation and may be expressed by using the $K_{m}$ Bessel function only, since perturbation should vanish at $r \rightarrow \infty$,

$$
\begin{aligned}
& u_{r}(r)= \pm B_{2} k K_{m}^{\prime}(s k r), \\
& p(r)=i \rho B_{2} \omega_{\mathrm{ext}}(r) K_{m}(s k r),
\end{aligned}
$$


where $s$ is the sign of the real part of $k$ to ensure the decrease toward zero at large $r, \omega_{\text {ext }}(r)$ is the frequency in the frame rotating and translating with the fluid at the location $r$ outside the core

$$
\omega_{\mathrm{ext}}(r)=\omega-m \frac{\Delta \Omega R^{2}}{r^{2}}-U_{\infty} k
$$

and $B_{2}$ is a constant. As mentioned in Sec. II B, the decrease at large $r$ is exponential with a typical decrease-rate equal to $\sqrt{k^{2}+m^{2} / R^{2}}$ for $k R$ and $m$ sufficiently large.

c. Jump conditions. The inner and outer solutions have to satisfy two jump conditions. The kinematic condition imposes

$$
\frac{u_{r}\left(R^{-}\right)}{\omega_{j}}=\frac{u_{r}\left(R^{+}\right)}{\omega_{\mathrm{ext}}\left(R^{+}\right)}=i \eta
$$

where $\eta$ is the amplitude of the perturbation of the interface position relative to the cylindrical surface $r=R$ at equilibrium. The general dynamical condition enforces the continuity of pressure,

$$
\overbrace{\left[\partial_{r} P\left(R^{+}\right)-\partial_{r} P\left(R^{-}\right)\right]}^{\text {centrifugal }} \eta+\overbrace{p\left(R^{+}\right)-p\left(R^{-}\right)}^{\text {axial+ azimuthal shear }}=0 .
$$

The mean pressure gradient term $\left[\partial_{r} P\left(R^{+}\right)-\partial_{r} P\left(R^{-}\right)\right]$is associated with the centrifugal instability, whereas the perturbation pressure jump $p\left(R^{+}\right)-p\left(R^{-}\right)$leads to the $\mathrm{K}-\mathrm{H}$ instabilities. With the notation $\omega_{+}=\omega_{\text {ext }}\left(R^{+}\right)$for the frequency in the frame rotating with the outside fluid at $r=R$, we obtain

$$
\begin{aligned}
& \omega_{j} B_{2} s k K_{m}^{\prime}+i \frac{\omega_{+} k^{2}}{\rho \omega_{j} \beta^{2}} A_{1}\left(-\frac{2 m \Omega}{\omega_{j} r} J_{m}+\beta J_{m}^{\prime}\right)=0, \\
& i \frac{s B_{2} k K_{m}^{\prime}}{\omega_{+}} \rho\left(\Delta^{2} \Omega^{2} R-\Omega^{2} R\right)+i \rho \omega_{+} B_{2} K_{m}-A_{1} J_{m}=0 .
\end{aligned}
$$

This yields the dispersion relation:

$$
\begin{aligned}
& \left(\omega_{+}^{2}+s k R \frac{K_{m}^{\prime}(s k R)}{K_{m}(s k r)}\left(\Delta^{2} \Omega^{2}-\Omega^{2}\right)\right) \\
& \quad \times\left(-\frac{2 \Omega m}{\omega_{j}}+\beta R \frac{J_{m}^{\prime}(\beta R)}{J_{m}(\beta R)}\right)=-\frac{\omega_{j}^{2} R^{2} \beta^{2}}{s k r} \frac{K_{m}^{\prime}(s k R)}{K_{m}(s k r)},
\end{aligned}
$$

identical to the relation found by Lim and Redekopp. ${ }^{31}$ It is convenient to express the dispersion relation in terms of nondimensional parameters by introducing $R$ and $\Delta_{U}$ as length and velocity scales. Without a change of notation, we find

$$
\begin{aligned}
& ((\omega_{j}+\overbrace{k}^{\text {axial shear }}+\overbrace{m S(1-\Delta)}^{\text {azimuthal shear }})^{2}+\overbrace{s k \frac{K_{m}^{\prime}(s k)}{K_{m}(s k)} S^{2}\left(\Delta^{2}-1\right)}^{\text {centrifugal }}) \\
& \times(\overbrace{-2 m S+\omega_{j} \beta \frac{J_{m}^{\prime}(\beta)}{J_{m}(\beta)}}^{\text {core effect }})+\frac{\beta^{2} \omega_{j}^{3}}{s k} \frac{K_{m}^{\prime}(s k)}{K_{m}(s k)} \\
& =D[k, \omega, m, S, \Delta]=0,
\end{aligned}
$$

with the new nondimensional variables

$$
\begin{aligned}
& \omega_{j}=\omega-m S-(1+a) k, \\
& \beta=k \sqrt{\frac{4 S^{2}-\omega_{j}^{2}}{\omega_{j}^{2}},}
\end{aligned}
$$

where $a, S$, and $\Delta$ have been defined previously. In order to simplify the physical interpretation of the dispersion relation, the origin of each term has been labeled in Eq. (21), traced back from the kinematic (17) or dynamic (18) jump conditions.

\section{TEMPORAL INSTABILITY}

The next two sections deal with the temporal stability problem. We assume $k$ to be real and solve the dispersion relation for a complex frequency $\omega$. The dependence on external flow parameter $a$ manifests itself as a simple Doppler frequency shift, and we therefore set $a=0$ for the temporal stability analysis. On the other hand, if $k$ is complex (as assumed for the spatial instability theory), the advection parameter $a$ is of fundamental importance.

\section{A. Asymptotic results of the temporal instability analysis}

To understand the interplay of the axial and azimuthal shear, the centrifugal instability and the effect of the core, we expand the dispersion relation both in the limit $k \gg 1$ and $k$ $\ll 1$. As the reader will see, the $k \gg 1$ limit will confirm the physical considerations presented in Sec. II B.

The procedure is as follows: since the magnitude of $\beta$ $=k \sqrt{\left(4 S^{2}-\omega_{j}^{2}\right) / \omega_{j}^{2}}$ with respect to $k$ is unknown, we assume $\beta$ a priori to be large, order unity or small, then derive the asymptotic expression for the dispersion relation, compute the solution $\omega$ as a function of $k$ and finally verify the validity of the previous assumptions on the size of $\beta$. As was already mentioned, although we are mostly interested in results for $\Delta=0$, we still treat the general case to identify precisely the different dominant terms.

\section{High wave number regime $k \gg 1$}

Assuming $\beta \ll k$, the only solution is a neutral mode given to leading order by

$$
\omega=(m+2 \alpha) S+(1+a) k,
$$

where $\alpha$ is the sign of $m$. This asymptotic formula appears to be an exact solution of the dispersion relation. It corresponds 
to $\beta=0$ and represents a Kelvin wave of frequency $\omega_{j}$ $=2 \alpha S$, with phase planes normal to the axis of rotation.

Assuming $\beta \sim k$, we get an unstable wave with

$$
\omega \sim\left(\frac{1 \pm i}{2}\right) k+a k
$$

which corresponds to a Kelvin-Helmholtz mode associated with the axial shear. Note the existence of a pair of complexconjugate frequencies, with one unstable branch and one evanescent one. This is due to the inviscid character of our model. In what follows, only the unstable branch will be considered. The leading-order expression of this unstable mode does not involve $S$, confirming that, for any finite $m$, the axial shear dominates over other mechanisms at large $k$. To obtain the contribution of the azimuthal shear and centrifugal instability, we expand $\omega$ to the next order in $1 / k$, using the expansions for the Bessel functions

$$
\begin{aligned}
& \lim _{k \rightarrow+\infty} \frac{K_{m}^{\prime}(k)}{K_{m}(k)}=-1+\frac{1}{2 k}+O\left(\frac{1}{k^{2}}\right), \\
& \lim _{\beta \rightarrow \infty} \frac{J_{m}^{\prime}(\beta)}{J_{m}(\beta)}=-i-\frac{1}{2 \beta}+O\left(\frac{1}{\beta^{2}}\right),
\end{aligned}
$$

we get the following expression for $\Delta \neq 1$ :

$$
\begin{aligned}
\omega= & \frac{\overbrace{}^{\frac{1}{2}}[-m S \Delta+k]+a k+\frac{1}{2} i}{\text { mean advection }} \\
& \times[\overbrace{m S(1-\Delta)}^{\text {azimuthal shear }}+\overbrace{k}^{\text {axial shear }}+\overbrace{S^{2}\left(1-\Delta^{2}\right)}^{\text {centrifugal }}] .
\end{aligned}
$$

In expression (28), the real part of $\omega$ shows that the wave will be stationary in the frame moving at the mean speed $u_{z}=1 / 2$ in $r=1$ and rotating at the mean angular velocity $S \Delta / 2$. Using this asymptotic analysis, we recover the heuristic results of Sec. II B for the growth-rate of the "tilted shear instability" added with the centrifugal instability. The balance of the azimuthal and axial shear and centrifugal instabilities is now given by the imaginary part of Eq. (28). The axial shear gives a contribution to the growth rate equal to $k / 2$ since the jump in axial velocity is equal to unity in nondimensional variables, the azimuthal shear to $m / 2$ weighted by the jump in azimuthal velocity $(1-\Delta) S$. The centrifugal instability contribution is independent of both $k$ and $m$, as already discussed in Sec. II B and equals $S^{2}\left(1-\Delta^{2}\right) / 2$. When $m$ is positive, the azimuthal shear and the centrifugal effect cooperate to destabilize the flow, whereas when $m$ is negative a competition ensues, but except for $\Delta$ close to unity or large $S$, the azimuthal shear dominates and reduces the growth rate. More precisely, for $m<0$, when $S$ $<-m / 2(1+\Delta)$, an increase in swirl will have a stabilizing effect whereas when $S>-m / 2(1+\Delta)$, it will have a destabilizing effect. These effects were already discussed by physical arguments in Sec. II B and were attributed to the tilting of the shear with respect to the local wave number. We shall point out that, at high $k$, the instability is driven at leading order by the axial shear and at the next order by the azimuthal shear and the centrifugal instability.

\section{Low wave number regime $k \ll 1$}

a. $m=0$, bulging mode. The asymptotic analysis for the axisymmetric mode $m=0$ for $k \ll 1$ is not reproduced here but detailed in Appendix A. For $\Delta \neq 1$, the compatibility condition requires $\beta=O(1)$, which gives

$$
\omega_{0}=k(1+a)+i \frac{2 S}{Y_{\Delta}} k,
$$

where $Y_{\Delta}$ is the solution of Eq. (A3) which has to be determined numerically. This mode is stationary $\left(\omega_{r}=0\right)$ in a frame moving with the core and centrifugally unstable with a vanishing growth rate as $k \rightarrow 0$. This behavior is common among centrifugally unstable configurations such as TaylorCouette flow.

b. $m \neq 0$ : bending modes $(m= \pm 1)$ and spiral modes $(|m|>1)$. We present the results for $m \neq 0$ and assume $\beta$ $\ll 1$ since other assumptions on $\beta$ do not give rise to unstable branches. Using the expansions for the Bessel functions,

$$
\begin{aligned}
& \lim _{\beta \rightarrow 0} \beta \frac{J_{m}^{\prime}(\beta)}{J_{m}(\beta)}=|m|-\frac{\beta^{2}}{2|m|+1}+O\left(\beta^{4}\right), \\
& \lim _{k \rightarrow 0} k \frac{K_{m}^{\prime}(k)}{K_{m}(k)}=-1+O\left(k^{2}\right),
\end{aligned}
$$

the dispersion relation may be written to leading order in $k$ as

$$
\begin{gathered}
\omega_{j}^{2}+(\overbrace{\alpha S}^{\text {core }}+\overbrace{m S(1-\Delta)}^{\text {azimuthal shear }}) \omega_{j} \\
+\frac{\overbrace{m^{2} S^{2}(1-\Delta)^{2}}^{\text {azimuthal shear }}-\overbrace{|m| S^{2}\left(\Delta^{2}-1\right)}^{\text {centrifugal }}}{2}=0 .
\end{gathered}
$$

Stability is then determined by the sign of its discriminant $\delta$,

$\delta=S^{2}(\overbrace{1}^{\text {core }}-\overbrace{m^{2}(1-\Delta)^{2}}^{\text {shear }}+\overbrace{2|m|(1-\Delta)}^{\text {core }+ \text { shear }}-\overbrace{2|m|\left(1-\Delta^{2}\right)}^{\text {centrifugal }})$.

In Eq. (32) and Eq. (33), we have traced back the physical origin of various terms from Eq. (21). From Eq. (33) we clearly see that the core has a stabilizing effect, whereas the azimuthal shear and the centrifugal instability have a destabilizing effect. The larger $m$, the stronger the dominance of the shear instability, as expected from physical considerations given in Sec. II B.

In Fig. 6, we plot $\delta(m, \Delta)$ as a function of $\Delta$ for different values of $m$. For each $|m|>1$, there is a critical value of $\Delta$, $\Delta_{m}$, such that the mode is unstable when $\Delta<\Delta_{m}$ and neutral to order 0 in $k$ when $\Delta \geqslant \Delta_{m}$. The threshold $\Delta_{m}$ equals

$$
\Delta_{m}=\frac{m^{2}-m-\sqrt{2\left(m^{2}-m\right)}}{m^{2}-2 m}
$$

and grows with $m$. The value of $\Delta_{m}$ results from the interplay between the stabilizing effects of the core and the destabilizing effect of the azimuthal shear and the centrifugal force. For $m=1, \Delta_{1}=0$, the effects of azimuthal shear, centrifugal force, and core balance at leading order and it becomes necessary to go to higher order, as explained in Appendix B. It is found that stabilization occurs for large enough swirl. 


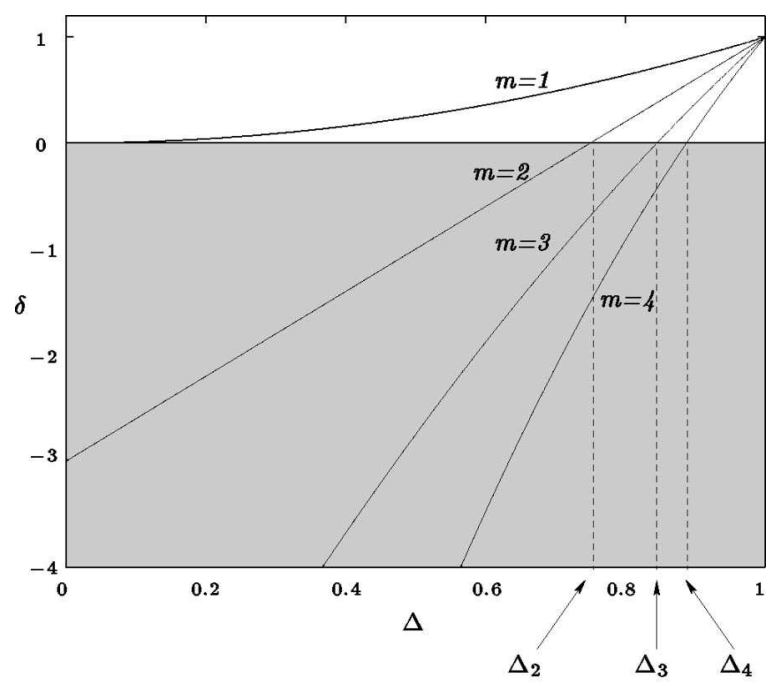

FIG. 6. We see $\delta$ (see the text) with respect to $\Delta$. The shaded region corresponds to instability.

For $m>1$, when $\Delta<\Delta_{m}$, i.e., when the jump in azimuthal velocity between the core and the surrounding fluid is large enough, the modes are unstable at $k=0$ with a finite growth rate that equals $\sqrt{-\delta} / 2$. Note that this growth rate is independent of the sign of $m$. When $\Delta=0$, the destabilizing centrifugal term is entirely balanced by a core term and $\sigma$ $=\sqrt{S^{2}\left(m^{2}-1\right)} / 2$ is solely due to an azimuthal shear instability weakened by a core effect. For large $m$, the $k=0$ growth rate may be approximated by $\sigma=|m| S / 2$, which is compatible with the physical prediction of Sec. II B 2 taking into account only the azimuthal shear.

At order 1 in the expansion of $\omega$ versus $k$ we obtain for the unstable mode, i.e., when $\Delta<\Delta_{m}$,

$$
\sigma_{\Delta<\Delta_{m}}=\frac{\sqrt{-\delta}\left(1+\frac{\alpha-m(1-\Delta)}{\delta} k S\right)}{2},
$$

where $\alpha$ is the sign of $m$. The sign of the slope of the growth rate at $k=0$ (the term $[\alpha-m(1-\Delta)] S / \delta$ in (35)) reflects the competition between azimuthal shear [the $-m(1$
$-\Delta) S / \delta$ ) term in (35)] and stabilization by the core [the $\alpha S / \delta$ term in (35)]. Note also that the modes $+m$ and $-m$ have the same growth-rate at $k=0$ but opposite slopes ${ }^{45}$ independent of $S$, since $\delta$ is proportional to $S^{2}$.

When $\Delta=0$, the screening is total (the outer fluid is not rotating) and the growth rate becomes

$$
\sigma=\frac{S \sqrt{m^{2}-1}}{2}+\left\{\begin{array}{l}
\frac{\sqrt{m^{2}-1} \frac{k}{m+1} \frac{1}{2},}{} \text { when } m \geqslant 2 ; \\
\frac{\sqrt{m^{2}-1} \frac{k}{m-1},}{2}, \text { when } m \leqslant-2 .
\end{array}\right.
$$

This results, when $m$ is large, in

$$
\sigma \sim \frac{1}{2}|m S+k|+R(m)+\mathcal{O}\left(\frac{k}{m}\right)
$$

where $R(m) \sim \mathcal{O}(1 / m)$ is a quantity that goes to zero when $m \rightarrow \infty$, independently of the value of $k$. If one is willing to neglect this correction with respect to terms of order $k$ in order to retain the first $k$ contribution, the prediction of the "tilted shear approximation" of Eq. (5) following the physical interpretation given in Sec. II B 2 is recovered. It is fully justified when $1 / m \ll k \ll 1$.

In conclusion, the behavior at small $k$ depends on the wave number $m$; for $m=0$, the instability is centrifugally driven and vanishes at $k=0$; for $m= \pm 1$, stabilization due to the core occurs at large enough swirl; for $|m|$ larger than 1, $k=0$ is unstable due to azimuthal shear and for $\Delta=0$, an increase of $k$ is destabilizing for positive $m$ and stabilizing for negative $m$, as explained by the "tilted shear" approximation.

The unstable modes predicted asymptotically are summarized in Table I for a completely screened vortex $(\Delta$

\begin{tabular}{|c|c|c|c|c|}
\hline$k$ & $\beta$ & $\begin{array}{c}m=0 \\
\text { Bulging mode }\end{array}$ & $\begin{array}{c}m= \pm 1 \\
\text { Bending modes }\end{array}$ & $\begin{array}{c}\quad|m|>1 \\
\text { Spiral modes }\end{array}$ \\
\hline$k \gg 1$ & $\beta \sim i k$ & & $\omega=\frac{k-m S}{2}+\mathbf{i} \frac{\left(\mathbf{k}+\mathbf{m S}+\mathbf{S}^{2}\right)}{\mathbf{2}}$ & \\
\hline \multirow[t]{2}{*}{$k \ll 1$} & $\beta \ll 1$ & & $\begin{aligned} \omega_{ \pm 1} \sim\left(a+\frac{1}{2}\right) k+\alpha \frac{S}{8} k^{2} \\
+\quad\left(\frac{\sqrt{1-\mathbf{S}^{2} / \mathbf{2}}}{2} \mathbf{k}+\alpha \frac{\mathbf{S}}{\mathbf{8} \sqrt{\mathbf{1 - \mathbf { S } ^ { 2 } / \mathbf { 2 }}} \mathbf{k}^{2}}\right.\end{aligned}$ & $\begin{array}{l}\omega_{m} \sim \frac{m-\alpha}{2} S+\left(a+\frac{1}{2}\right) k \\
\quad+\mathbf{i} \frac{\sqrt{\mathbf{m}^{2}-\mathbf{1}}\left(\mathbf{s}-\frac{\alpha-\mathbf{m}}{\mathbf{m}^{2}-\mathbf{1}} \mathbf{k}\right)}{\mathbf{2}}\end{array}$ \\
\hline & $\beta=O(1)$ & $\omega_{0} \sim(1+a) k+\mathrm{i} \frac{2 S}{Y_{0}} k$ & & \\
\hline
\end{tabular}
$=0)$.

TABLE I. Fully screened swirling jet $(\Delta=0)$; asymptotic expressions of the unstable branches of the dispersion relation; growth rates in bold. 


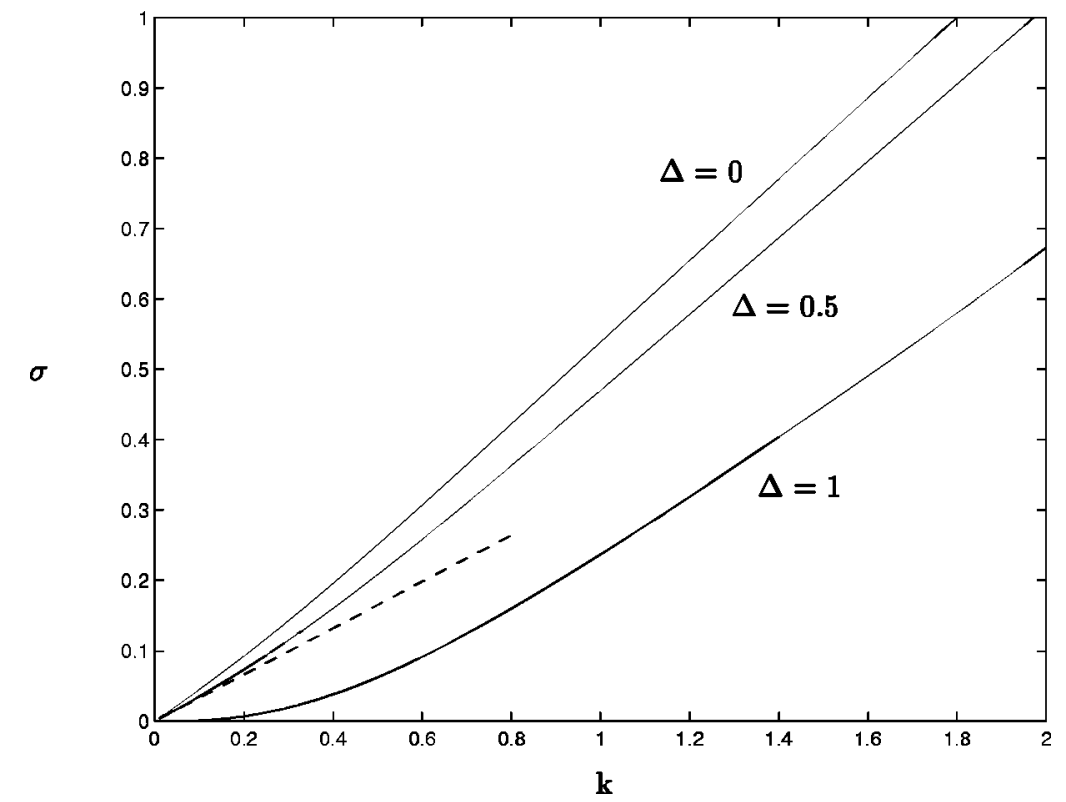

FIG. 7. Growth rate $\sigma=\omega_{i}$ of the axisymmetric mode $m=0$ for $S=1$ and $\Delta=0,0.5,1$; “--:" asymptotic expression at small $k$ obtained for $\Delta=0.5$.

\section{B. Numerical results for the temporal stability analysis}

In the preceding section we have used asymptotic expansions to trace the origin of physical mechanisms responsible for stabilization and destabilization of the base flow. We will now proceed to a more general analysis and determine the temporal instability branches of the dispersion relation. As the dispersion relation is transcendental, a numerical solution is necessary. At a given value of the swirl $S$, the screening parameter $\Delta$ and the azimuthal wave number $m$, we determine all the temporal branches $\omega(k)(k \in \mathbb{R})$, using a procedure described in LCH. The influence of the screening parameter $\Delta$ will be analyzed to provide a connection to the results of LCH (corresponding to $\Delta=1$ ).

\section{Axisymmetric perturbations: The bulging mode $m=0$}

Figure 7 presents the growth rate as a function of $k$ along the unstable branch for the axisymmetric mode $m=0$ at $S$ $=1$ and three different screening parameters $\Delta: \Delta=1$, the case considered by LCH, corresponding to the Rankine vortex, $\Delta=0.5$, corresponding to a jump in azimuthal velocity at $r=1$ of half the azimuthal velocity, and $\Delta=0$ corresponding to the fully screened Rankine vortex, where the azimuthal velocity jumps to zero at $r=1$. We observe that an increase in the azimuthal velocity jump promotes instability for all wave numbers $k$.

In Fig. 7, the asymptotic expression $\sigma_{\text {as }}$ valid for small $k$ derived in Appendix A is plotted as a dashed line for a partial screening $\Delta=0.5$, in excellent agreement with the numerical result. Also in excellent agreement are the asymptotic expressions (28) valid for large $k$, as shown in Table II for $S$ $=1$ and $\Delta=0.5$. The asymptotic expression derived in (28) is exact at order -1 as $k$ goes to infinity, with the error vanishing with $k$ at least as $1 / k$. Not only the relative error is vanishing but also the absolute error $\left|\sigma_{\text {as }}-\sigma_{\text {num }}\right|$.

Figure 8 presents the evolution of the growth rate $\sigma$ with respect to the wave number $k$ as the swirl is increased from $S=0$ to $S=2$ in steps of 0.5 for a fully screened vortex ( $\Delta$ $=0$ ). The swirl has a destabilizing effect for all wave numbers $k$, as predicted by the asymptotic expressions derived for small and large $k$ (Table I). Since the azimuthal wave number $m$ equals zero, the azimuthal shear does not play any role in the destabilization. At large $k$, expression (28) and the results plotted in Fig. 8 show that the $\mathrm{K}-\mathrm{H}$ instability growth rate due to the axial shear (independent of rotation) is augmented by a centrifugal term. For small $k$, the expression derived in Appendix A results from a balance of axial shear, centrifugal and core forces. The numerical results confirm that, for all $k$, the stabilizing role of the core is overwhelmed by the destabilizing action of the centrifugal force. By contrast, for $\Delta$ $=1, \mathrm{LCH}$ observed a stabilizing effect of the swirl, for all $k$, since in that case there is no centrifugal instability, and the only effect is a stabilization due to the core.

\section{Large $m$ (i.e., $m \geqslant 2)$ : Spiral modes}

All azimuthal wave numbers $m$, except the bending modes $m= \pm 1$ and the bulging mode $m=0$ behave in a

TABLE II. $\Delta=0.5 ; S=1$; asymptotic expressions at high $k$ compared to numerical values; $\sigma_{\text {as }}$ is the value of the growth rate $\sigma$ obtained from the asymptotic expression (28) and $\sigma_{\text {num }}$ the numerically computed value of $\sigma$.

\begin{tabular}{ccccrrr}
\hline \hline$k$ & 3 & 5 & 10 & 20 & 50 & 100 \\
\hline$\sigma_{\text {as }}$ & 1.87 & 2.87 & 5.37 & 10.37 & 25.37 & 50.37 \\
$\sigma_{\text {num }}$ & 1.57 & 2.66 & 5.26 & 10.31 & 25.34 & 50.36 \\
$\sigma_{\text {as }}-\sigma_{\text {num }}$ & 0.3 & 0.21 & 0.11 & 0.06 & 0.03 & 0.01 \\
\hline \hline
\end{tabular}




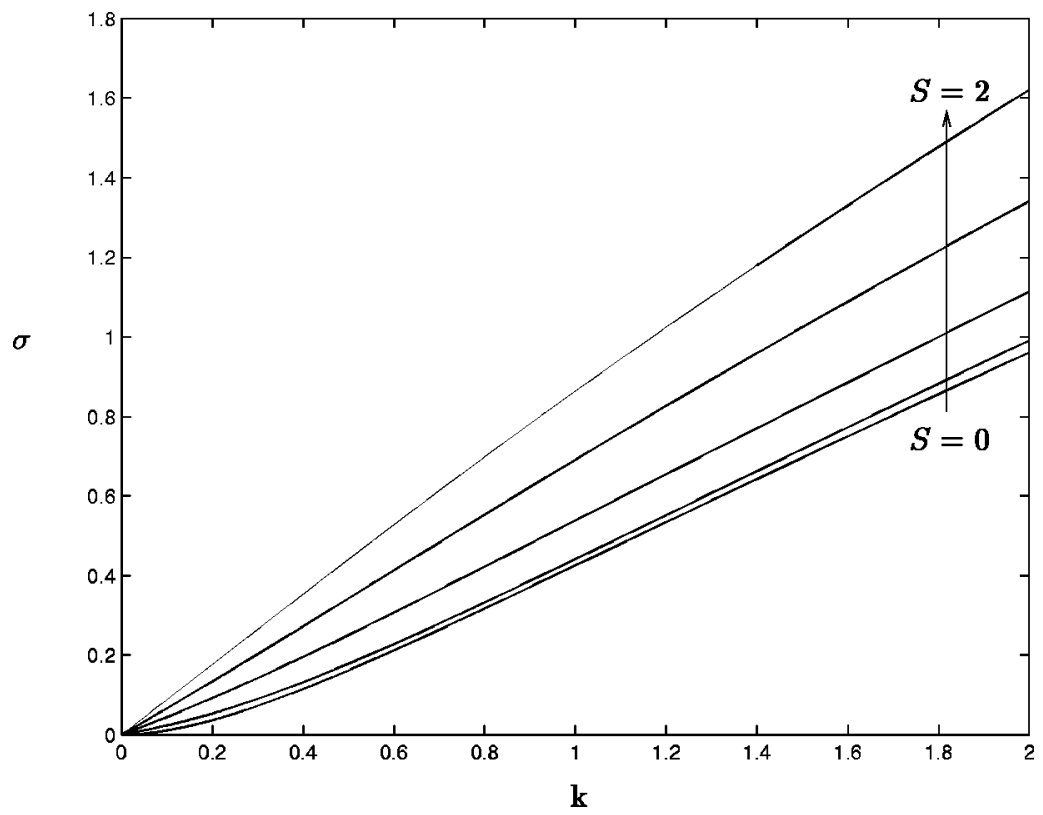

FIG. 8. Computed growth rate $\sigma$ of the $m=0$ axisymmetric mode for various $S(=0,0.5,1,1.5,2)$ and $\Delta$ $=0$. similar manner. Although results have been produced systematically for $m= \pm 2, m= \pm 3, m= \pm 4$, and $m= \pm 5$, we will illustrate the general behavior on the case $m= \pm 3$. Figure 9 is similar to Fig. 7, except that the swirl has been chosen equal to 0.3 ; in addition, we plot four values of $\Delta$. The effect of increasing the screening (decreasing $\Delta$ from 1 to 0 ) is destabilizing at small $k$ for both $m=+3$ and $m$ $=-3$, whereas at large $k$ it promotes instability for $m=+3$ and reduces instability for $m=-3$.

At large $k$, this result can be interpreted using the asymptotic results (28). Two terms are added to the axial Kelvin-Helmholtz growth rate $k / 2$, the centrifugal term equal to $\left(1-\Delta^{2}\right) S^{2} / 2$ and the azimuthal Kelvin-Helmholtz term $m S(1-\Delta) / 2$. As predicted by the "tilted shear" approximation, when $m$ is positive both terms conjugate their destabilizing effect whereas when $m$ is negative the azimuthal $\mathrm{K}-\mathrm{H}$ term is stabilizing and the centrifugal term remains destabilizing. For $(1+\Delta) S<-m$ (which is verified

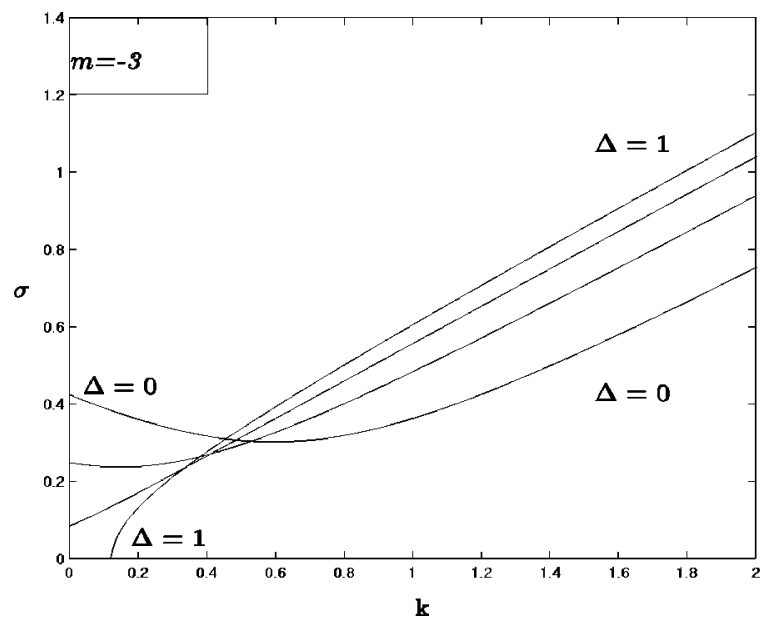

for the present choice of parameters) the azimuthal shear dominates and reduces the large- $k$ growth rate for negative $m$, as observed in Fig. 9(a).

At small $k$, for both $m=+3$ and $m=-3$, the growth rate becomes non-zero at $k=0$ as long as $\Delta<\Delta_{m}$ which is in perfect agreement with the asymptotic prediction. As pointed out there, $\Delta_{m}$ reflects the balance of the destabilizing azimuthal shear and the stabilizing Coriolis forces in the core.

Figure 10 is similar to Fig. 8. The temporal instability branch is plotted for various swirls and for a total screening $(\Delta=0)$. For positive helical modes $m=+3$ it is shown that the swirl promotes instability at all $k$, whereas for negative helical modes $m=-3$, one must consider two regimes of $S$ (see Sec. II A 1). In a first regime $S \leqslant-m / 2$, the swirl is destabilizing at small $k$ and stabilizing at large $k$. In a second regime $S \geqslant-m / 2$, the swirl is destabilizing for all $k$. Figure 10(a) exemplifies the role of the swirl in the first regime. For small swirl levels $(S \leqslant|m| / 2)$, the growth rate is mainly

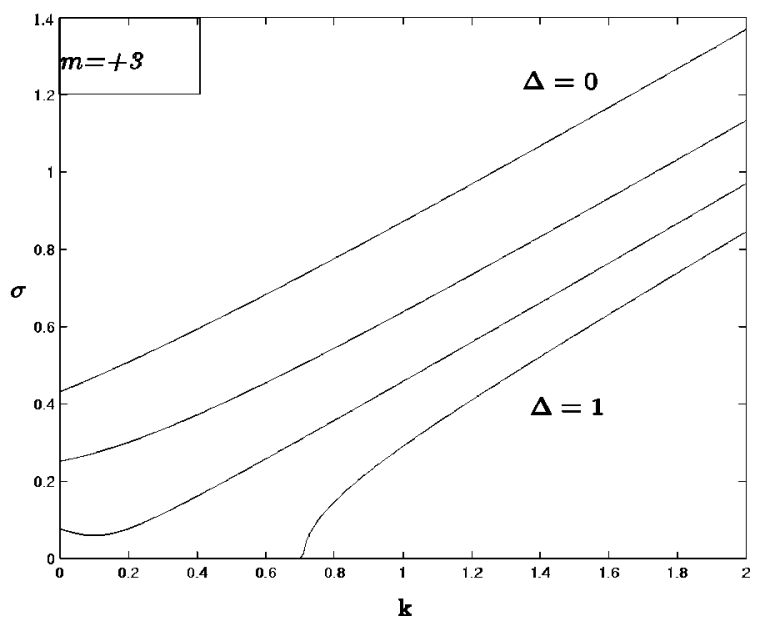

FIG. 9. Growth rate $\sigma$ of $m=-3$ and $m=+3$ for $\Delta=0,0.5,0.8,1$, and $S=0.3$. 


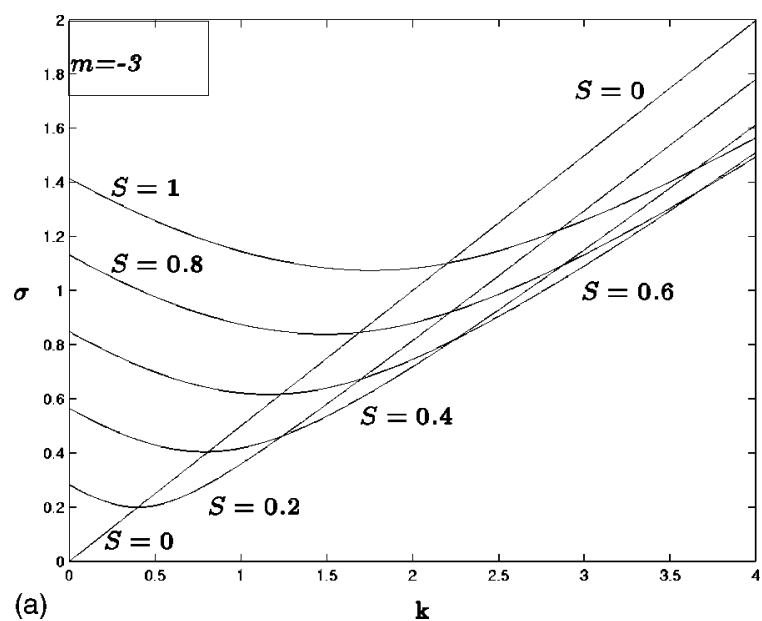

FIG. 10. Growth rate $\sigma$ of $m=-3$ and $m=+3$ for various $S=0,0.2,0.4,0.6,0.8,1$, and $\Delta=0$. Please note the different scaling on the $k$ axis.

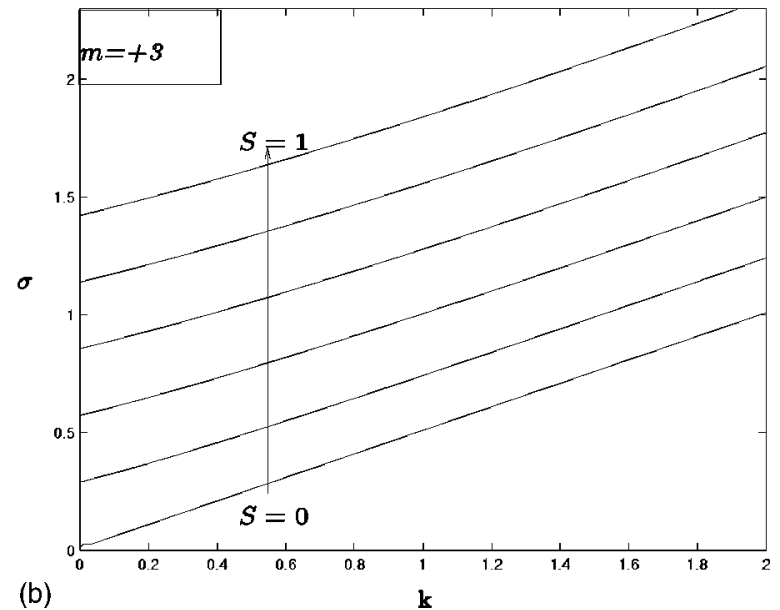

(b) given by the $\mathrm{K}-\mathrm{H}$ instability due to the "tilted shear" since the contribution of the azimuthal shear is proportional to $m S$, whereas the centrifugal instability is proportional to $S^{2}$.

At $k=0$ both helical waves $m=+3$ and $m=-3$ have opposite slope and the same growth rate, which is directly proportional to the swirl $S$. These characteristics are predicted by the asymptotic calculation, Eq. (36). As was pointed out there, the growth rate at $k=0$ is entirely given by the azimuthal shear with a small correction due to the action of the core that vanishes as $m$ increases. The slope at the origin $-\left[(\alpha-m) / 2 \sqrt{m^{2}-1}\right] k$ renders the effect of the "tilted shear" that destabilizes positive azimuthal wave numbers and stabilizes negative ones. Let us emphasize that for total screening $(\Delta=0)$, the effect of the centrifugal instability at small $k$ is entirely balanced by the stabilizing role of the core, in which case only the shear contributes to the instability.

In order to probe the relevance of the simple "tilted shear" model at large $m$, we have plotted in Fig. 11, for $m$
$=+3$ and $m=-3, \Delta=0$ and $S=0.5$, the computed growth rates and the prediction given by Eq. (5), taking into account only the shear instability: $\sigma=1 / 2|m S+k|$. This figure, plotted for an intermediate value of swirl $(S=0.5)$ smaller than the value $S=|m| / 2$ ( $S=1.5$ presently), where centrifugal instabilities take over, shows that the "tilted shear layer," combining axial and azimuthal shear, is a good approximation. At small $k$, the centrifugal instability contribution is balanced by the core contribution and at large $k$, it is smaller than the azimuthal shear contribution since the former varies like $S^{2}$ and the latter like $m S$.

\section{Bending waves: $m= \pm 1$}

Last but not least, bending modes $m= \pm 1$ are discussed. Since their dynamics is more intricate, we solely focus on the complete screening of the vortex, i.e., $\Delta=0$. Figure 12 is similar to Fig. 10 and presents the value of the growth rate as a function of $k$ for $m=+1$ and $m=-1$ and $\Delta=0$. At high $k$,

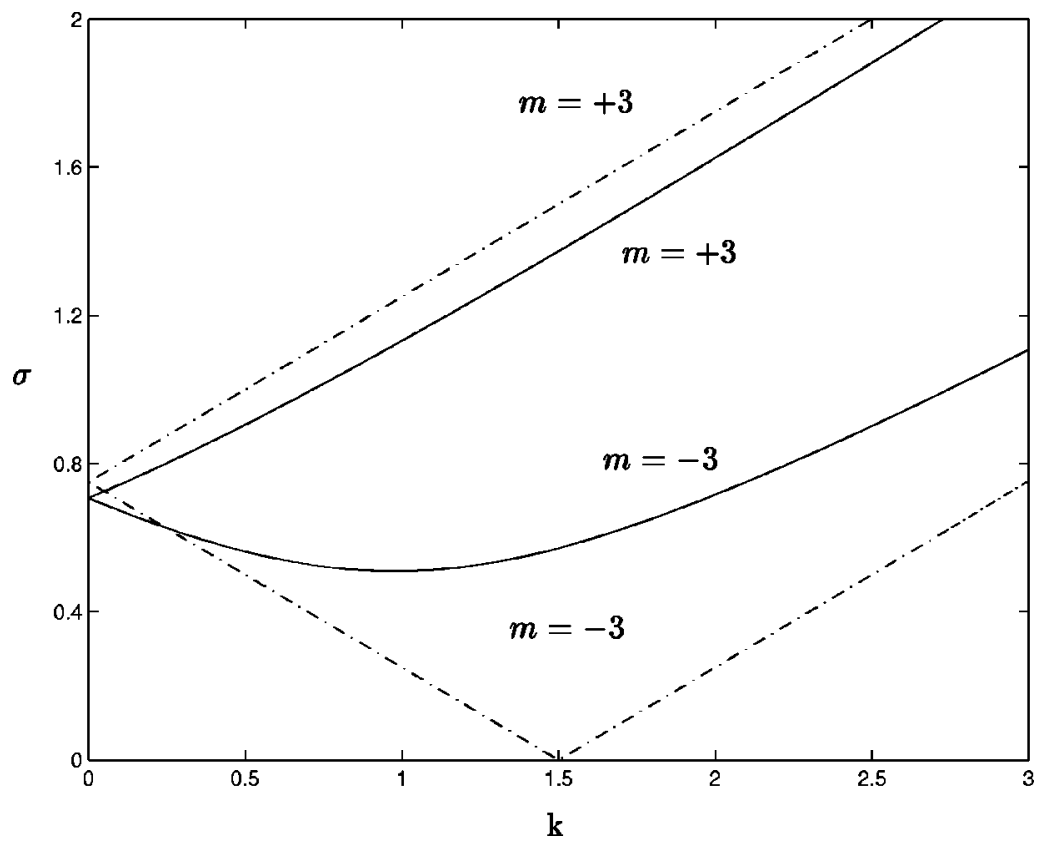

FIG. 11. Growth rate $\sigma$ of $m=-3$ and $m=+3$ for $S$ $=0.5$ and $\Delta=0$; "tilted shear" prediction (-.-). 

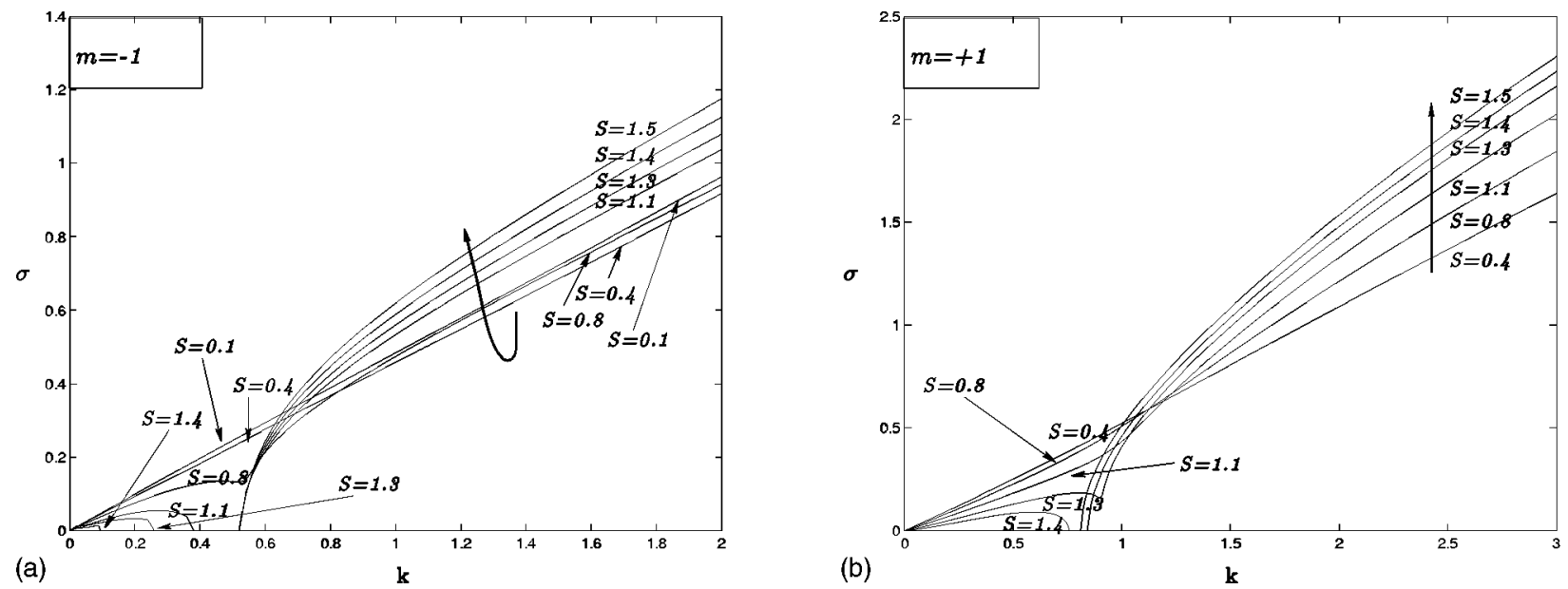

FIG. 12. Growth rate $\sigma$ of $m=-1$ (a) and $m=+1$ (b) for various $S$ and $\Delta=0$.

for $m=-1$, expression (28) predicts that an increase in swirl is destabilizing for $S>-m / 2(S>0.5$ presently) and stabilizing for $S<0.5$. Indeed, in Fig. 12(a), at large $k$, the curve for $S=0.1$ and $S=0.8$ are above the one for $S=0.4$. At small $k$, the swirl has a stabilizing effect. We must distinguish between three swirl ranges. We see in Fig. 12(a) the existence of a threshold value $S_{-1}$ determined numerically as 0.95 . For $S \in\left[0 ; S_{-1}\right]$, the flow is unstable for all $k$, and increasing $S$ in this range induces a stabilization for low $k$. In a second regime, $S \in\left[S_{-1} ; \sqrt{2}\right]$, the flow becomes neutral for wave numbers $k$ in $\left[k_{-1}^{\text {inf }}, k_{-1}^{\text {sup }}\right]$, where $k_{-1}^{\text {inf }}$ decreases with increasing swirl and $k_{-1}^{\text {sup }}$ is close to 0.5 . In the last regime, $S$ $\geqslant \sqrt{2}$, the complete stabilization occurs from $k_{1}^{\text {inf }}=0$ to $k_{-1}^{\text {sup }}$ $\sim 0.53$. The asymptotic calculation pushed at second order in the limit $k \rightarrow 0$ (which is presented in Appendix B) predicts this critical value of $S=\sqrt{2}$, beyond which small wave numbers $k$ are stabilized, and links this effect to the role of the core.

Figure 12(b) is similar to Fig. 12(a), but for $m=+1$. At large $k$, it shows that the swirl destabilizes the flow, again in agreement with the asymptotic result, since for $m$ positive both the azimuthal shear and the centrifugal instability are destabilizing. At small $k$, we define from systematic numerical computations three swirl regimes as we did before: the behavior is similar to the $m=-1$ case, a first regime $S$ $<S_{+1} \sim 1.35$ where all wave numbers $k$ are unstable, a second regime $S_{+1}<S<\sqrt{2}$, where a neutral region $\left[k_{+1}^{\text {inf }}, k_{+1}^{\text {sup }}\right]$ of the $k$ axis appears, and a third regime $S>\sqrt{2}$, where small wave numbers are entirely stabilized. Asymptotic expressions for $k \rightarrow 0$, derived in Appendix B, again predict the existence of a critical swirl $S=\sqrt{2}$ beyond which small wave numbers are entirely stabilized.

\section{CONVECTIVE/ABSOLUTE INSTABILITY TRANSITION}

The spatiotemporal study developed next analyzes the effect of the centrifugal force and azimuthal shear on the propagation of the instabilities, whereas temporal instability previously discussed addresses solely their effect on the growth rate. In swirling jets or swirling flows in pipes, per- turbations are continuously injected into the flow at the inlet and one may wonder what will be their fate. Furthermore, control of the flow, in particular jet flow, is often achieved by acoustic means, with acoustic waves being transformed into vortical perturbations at the nozzle where the receptivity is the highest. The response to these perturbations depends on how they are advected and amplified by the flow. Classically $^{36}$ this leads to the distinction between absolute and convective instability and readers are referred to Huerre and Monkewitz ${ }^{36}$ for details.

If the flow is convective (C), its response to a forcing will be given by spatial theory, which uses solutions of the dispersion relation for $\omega$ real and $k$ complex and associates with them an upstream or downstream energy propagation.

If the flow is absolute (A), self-sustained oscillations will overcome the response to small forcing amplitudes and knowing the response to a forcing requires the solution of the full global problem, including inlet and outlet conditions.

This study is a continuation of the study by LCH on the Rankine vortex with plug flow and also of the studies of Olendraru et al., ${ }^{25}$ Delbende et al., ${ }^{26}$ and Yin et al. ${ }^{27}$ on the Batchelor vortex, and finally of Lim and Redekopp ${ }^{31}$ and Loiseleux et al. ${ }^{30}$ on the Caflish vortex. Furthermore, Lim and Redekopp ${ }^{31}$ have already addressed the A/C nature of the instability of the screened Rankine vortex but have restricted themselves to axisymmetric perturbations $(m=0)$. From these studies, the general trend is that rotation favors the onset of absolute instability of both wakes and jets, but that the selection of the azimuthal wave number of the first mode to become absolute as the swirl is increased depends on the detail of the velocity profiles considered. Results of the present analysis will confirm this trend.

The procedure that we use is based on the determination of saddle points of the dispersion relation by looking at pinching points of the generalized spatial branches as described in LCH. Let us simply recall that the transition between convective and absolute instability is determined, for given $S$ and $m$, by varying $a$ until the Briggs-Bers criterium $^{37}$ is met. This determines two critical advection parameters, $a_{c}^{m}(S)$, as a function of $S$ and $m$, and at each of 


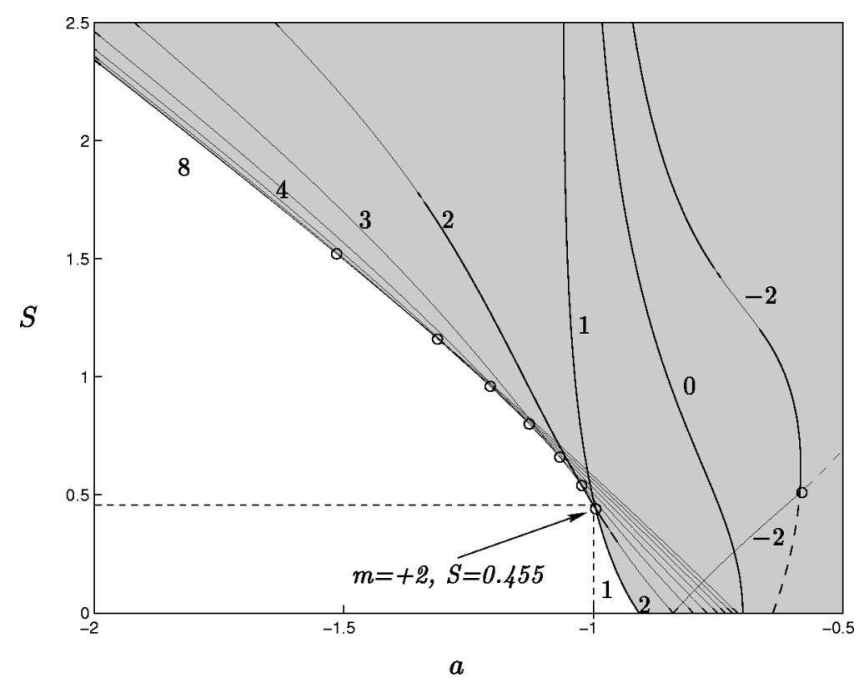

FIG. 13. Domains of absolute and convective instability with respect to the advection parameter $a$ and the swirl $S$. Each curve corresponds to the limit of the absolute instability domain for a particular $m$ (the absolute region being on the right of each curve).

these transitions, a real frequency $w_{0 r}$, called the absolute frequency, and a complex wave number $k_{0 r}+i k_{0 i}$ are selected.

By convention, the first transition that occurs for $a$ $<-1 / 2$ is called the wake $\mathrm{A} / \mathrm{C}$ transition since profiles with such a mean flow resemble wake profiles; the second transition for $a>-1 / 2$ is called the jet transition.

For jets with swirl $S$, each mode $m$ induces an A/C transition at the critical value $a_{c}^{m}(S)$, and the azimuthal wave number $m$ associated with the largest $a_{c}^{m}$ is called the transitional $m$, with its associated $a_{c}^{m}(S)$ called the transitional advection parameter $a_{c}(S)$. When $a_{c}(S)$ is negative, a certain amount of counterflow is necessary to trigger an absolute instability, whereas when $a_{c}(S)$ is positive the instability is absolute, even with a coflow $a$ if $a<a_{c}$. The value of $S$ such that $a_{c}(S)=0$ is of particular importance as it corresponds to the absolute instability transition for a rotating jet with no outer flow.

Wakes are described in the same manner, but the value of $a$ corresponding to zero outer flow is $a=-1, a_{c} \geqslant-1$ corresponding to wakes with coflow, and $a_{c} \leqslant-1$ corresponding to wakes with counterflow. From now on, we focus on the completely screened vortex, setting $\Delta=0$.

\section{A. Convective/absolute transitions in wakes}

For wakes, the domains of absolute and convective instability are shown in Fig. 13, where $a_{c}^{m}(S)$ is plotted for $m=0, m=1, \ldots, 8$ and $m=-2$. In the absence of swirl $(S$ $=0)$, the plug-flow coflowing wake is known to be convectively unstable for all $|m|$ (note that without swirl, there is no difference between $m$ and $-m$ since no particular axial direction is singled out by the base flow). The helical mode $|m|=1$ first becomes absolutely unstable, but still a counterflow is needed. As $S$ is increased, the amount of counterflow $1+a$ needed for absolute instability decreases very significantly for positive helical modes. By contrast, negative

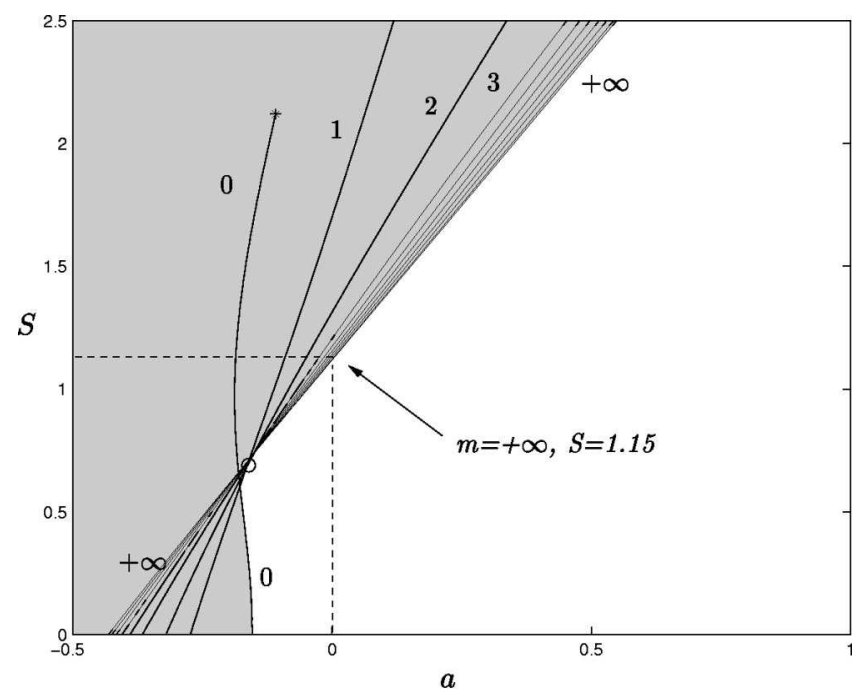

FIG. 14. Domains of absolute and convective instability with respect to critical advection parameter $a$ and swirl $S$ when $k$ is restricted to the halfplane $k_{r}>0$.

modes (the mode $m=-2$ is plotted as an example in Fig. 13) become less absolutely unstable. When $S>0.43$, the $m$ $=+2$ mode becomes "more absolute" than the $m=+1$ bending mode and this point is marked by a circle in Fig. 13. As soon as $S>0.455$, coflowing jets $(a \leqslant-1)$ become absolutely unstable to the $m=+2$ wave. The transitional azimuthal wave number remains equal to $m=+2$ until $S$ $=0.555$. It takes increasingly higher positive integer values, $m=+2, m=+3$, etc., before it eventually reaches $m=+8$ for $S>1.55$ which remains the transitional mode (i.e., the one being "the most absolute") for all $S>1.55$. These results clearly demonstrate the role of swirl in promoting the absolute instability of positive helical modes in agreement with the Loiseleux et al. ${ }^{30}$ study of the Caflish vortex, where the transitional modes were positive for the wake. But contrary to their findings this effect is in our case attributed not only to centrifugal instabilities but also to the azimuthal shear. This is in contrast to the studies of the Rankine vortex by $\mathrm{LCH}$ and the studies of the Batchelor vortex by Delbende et $a l .{ }^{26}$ and Olendraru et al. ${ }^{25}$ all showing that the negative helical modes and, in particular, the $m=-1$ mode are the critical ones.

\section{B. Convective/absolute transition in jets}

Figure 14 considers only convective/absolute transitions with $k_{0 r}>0$, along the line of Lim and Redekopp. ${ }^{31}$ Figure 14 is similar to Fig. 13, i.e., $a_{c}^{m}(S)$ is plotted versus $S$ for $m=0,1,2, \ldots, 10$ and 50 , with all larger $m$ behaving accordingly. Therefore $m=50$ is labeled $m=+\infty$ in Fig. 14. Negative helical modes have been found to be less absolutely unstable than their positive counterparts and are, for the sake of clarity, not plotted here. For $S=0$, the plug-flow jet without outer flow is convectively unstable for all $|\mathrm{m}|$. A certain amount of counterflow is necessary to trigger absolute instability, and the transitional mode is $m=0$ (see LCH for details). As soon as we introduce swirl, the amount of counterflow needed for transition for all $m$ diminishes, except for 
$m=0$. For $S=0.7$, all modes $m \geqslant 1$ have the same critical advection parameter $a_{c}=-0.2$. This particular point is marked by a circle in Fig. 14. For $S>0.7$, the most unstable mode becomes $m=+\infty$, which is the first to produce a transition at zero outer flow for $S=1.15$. Note that the $m=0$ transition curve is terminated when $S=2.1$ is reached since the saddle point enters the left $k_{0 r}$ plane.

The case $k_{0 r}=0$ corresponds to an eigenfunction that does not vanish at infinity and cannot be normalized properly. As we further increase $S$, we have to use an analytic continuation of the dispersion relation to track this saddle point. The analytic continuation is obtained from dispersion relation (21) by replacing $s k$ ( $s$ is the sign of the real part of $k$ ) by $k$. If it is possible to track the saddle point into the left $k_{0 r}$ plane, such a saddle point will produce nonphysical eigenfunctions (i.e., nonvanishing as $r \rightarrow \infty$ ) of the impulseresponse problem associated with the dispersion relation (21) (see Huerre and Monkewitz ${ }^{38}$ ). Following Huerre and Monkewitz $^{38}$ or Lim and Redekopp ${ }^{31}$ we reject these unphysical saddle points. We believe that the above problems result from the discontinuous and singular base velocity profiles. As was pointed out by LCH, these types of velocity profiles are not causal, since disturbances with infinitely small wavelengths are infinitely amplified. The prerequisites for applying the Briggs-Bers ${ }^{37}$ criterion are therefore not satisfied (no contour in the $\omega$ plane lies above the temporal instability branch). Only numerical calculations on causal profiles could clarify this point; work in this direction is currently in progress.

\section{DISCUSSION AND CONCLUSIONS}

The temporal instability properties of the screened Rankine vortex with plug flow have been determined in great detail. This base flow, in comparison to other singular swirling jet profiles, combines in the same model the four physical mechanisms that actually come into play in real flows: a core in solid body rotation guiding inertial waves, axial and azimuthal shear and centrifugal effects. This model is not fully realistic yet, since it lacks accounting for a finite shear layer thickness. Although we hope to address the effect of a finite shear layer thickness rigorously in forthcoming contributions, it will be discussed at the end of the present section based on physical arguments. The generality of the present results will then be addressed. Despite the above-mentioned limitations, the screened Rankine model is worth being considered since the analytical derivations allow a precise analysis of the stabilizing and destabilizing effects. The stabilizing role of the core is, in particular, identified and we propose an underlying physical mechanism that may also account for the results of $\mathrm{LCH}$. Furthermore, at small $k$, for helical modes with $|m| \geqslant 2$, the centrifugal destabilizing effect is entirely balanced by the core stabilization, and at $k=0$ the instability is entirely due to the azimuthal shear, as already noticed by Carton and McWilliams. ${ }^{4}$ At small $k$, the next order in the growth rate takes into account the axial shear and induces a decrease when $m$ is negative and an increase when $m$ is positive as predicted by the "tilted shear" estimate (5). At large $k$, the instability is due to the axial shear enhanced by cen- trifugal effects. It is further increased by the azimuthal shear when $m$ is positive or reduced by the azimuthal shear when $m$ is negative, in accordance with the "tilted shear" model.

For bending modes $(m= \pm 1)$, an increase in swirl stabilizes the $m= \pm 1$ modes at small $k$ due to the interaction with the core, but a destabilizing action at large $k$ under the action of azimuthal shear and centrifugal instability is observed. Finally, the bulging mode $m=0$ mode is destabilized both by the centrifugal instability and the axial shear for all $k$.

The question of interpreting the vortex breakdown by an $\mathrm{A} / \mathrm{C}$ transition can be addressed in two distinct ways. The first one relates the appearance of structures in the stagnation zone to the absolute nature of the wake behind the stagnation point. In this approach, the consideration of a Batchelor vortex seems relevant and recent studies of Yin et al. ${ }^{27}$ showed good agreement with experimental measurements. Following the second route, $\mathrm{LCH}$ tried to explain the appearance of vortex breakdown by an $\mathrm{A} / \mathrm{C}$ transition in the swirling jet as a natural generalization of the concept of a subcritical/ supercritical state that was introduced by Squire $^{39}$ and Benjamin. ${ }^{40}$ In our context, we believe that singularities in the modified Rankine vortex prevent any definite conclusions, since $m=\infty$ is the first mode to become absolutely unstable. Numerical simulations of a realistic profile using the technique of Delbende et al. ${ }^{26}$ are in progress to circumvent this unphysical property. In contrast, no difficulties have been encountered concerning the determination of saddle points on the wake side and it has been shown that azimuthal shear and centrifugal instability promote positive helical modes at the onset of absolute instability, in both wakes and jets. Helical structures are observed in the swirling jets before breakdown (see Billant et al. $)^{19}$ and in the wake of the vortex breakdown.

The essential question that should be addressed now is the generality of results obtained on model profiles and their applicability to experimental profiles where sharp gradients have been replaced by discontinuities. Although in some classical cases, like the axisymmetric jets and wakes ${ }^{41}$ the use of discontinuous profiles was successful in predicting the realistic absolute/convective thresholds, it is also known that a change in the shape of the profiles may radically alter the absolute/convective nature of the instabilities, as exemplified by the mixing layer (Huerre and Monkewitz ${ }^{38}$ ) when comparing results for the tangent hyperbolic profiles and the broken line profiles $\left(\right.$ Balsa $\left.^{42}\right)$.

Temporal stability studies are believed to be more robust than the spatiotemporal ones to modifications of the velocity profiles. The influence of a finite but small shear layer thickness on the temporal stability properties of mixing layers, jets, and wakes introduces a cut-off wave number scaling like the inverse of the shear-layer thickness on the instability curves obtained considering discontinuous profiles. In circular geometry, the wave number is two-dimensional and the cut-off should be based on the square of the total wave number $k^{2}+m^{2}$. At small $k$ and $m$, results of the present study should therefore extrapolate to realistic velocity profiles. In particular, the stabilizing role of the core for $m= \pm 1$ and small $k$ due to the coupling with Kelvin waves described in 
the present model should be generic to flows with KelvinHelmholtz instability and a rotating core. It is also active in the $\mathrm{LCH}$ model but not in the Caflish model ${ }^{29}$ because of the absence of a core in solid body rotation. The destabilization of azimuthal modes close to $k=0$ due to the azimuthal shear is also coherent with results for the 2-D stability of the realistic vortices of Carton and McWilliams ${ }^{4}$ or Carnevale and Kloosterziel, ${ }^{5}$ who have indeed shown that, as the azimuthal shear thickness becomes thinner, the most unstable azimuthal wave number becomes larger. For the 2-D flow, the most unstable mode is two dimensional and the cutoff imposed by the finite thickness of the azimuthal shear stabilizes the high azimuthal wave numbers $m$. The novelty here is that when a swirling jet is considered, the shear layer is tilted. For modes with $\sqrt{k^{2}+m^{2}}$ large but smaller than the cutoff $1 / \delta$, the inverse of the shear layer thickness, the present model shows that the total shear dominates and the most unstable modes in this range correspond to positive spirals, since the total wave number is then aligned with the local shear. When 1 $\ll \sqrt{k^{2}+m^{2}} \ll 1 / \delta$, the present study shows that the centrifugal effect varies like $S^{2}$ and is small compared to the shear effect that varies like $k+m S$.

When $m$ is larger or of order $1 / \delta$ the present model stops to be valid and one should refer to Ludwieg ${ }^{16}$ and Leibovich and Stewartson ${ }^{17}$ to understand the stability properties. These authors have revealed a general physical mechanism active in a broad variety of swirling jets and capable of destabilizing helical modes of high negative azimuthal wave numbers. Ludwieg ${ }^{16}$ considered perturbations localized at a prescribed radius and explained the underlying physical reason for the instability that should be interpreted as a centrifugal instability in a sloped reference frame aligned with the direction of vanishing strain. Leibovich and Stewartson ${ }^{17}$ performed a rigorous asymptotic expansion at large $k$ and $m$ with $m / k$ of order one and showed that, at any given radius $r_{0}$, it is possible to construct an eigenmode centered in $r_{0}$ with a maximum growth rate, at leading order in $1 / \sqrt{m}$, reached for the $(k, m)$ wave number pairs such that

$$
\beta=-k / m=\left.\frac{D\left(U_{\theta} / r\right)}{D U_{z}}\right|_{r_{0}},
$$

where $D$ stands for $d / d r$ and $\beta=-k / m$ is the helix angle. At any radius $r_{0}$, it is then possible to let $k$ and $m$ both go to infinity with $\beta=-k / m$ fixed and determined by Eq. (38). According to Leibovich and Stewartson, ${ }^{17}$ the eigenmode gets more and more localized at $r_{0}$ and the growth rate $\sigma\left(r_{0}\right)$ then asymptotes a constant,

$$
\sigma^{2}\left(r_{0}\right)=\left.\frac{2 U_{\theta}\left(r D U_{\theta}-U_{\theta}\right)\left(U_{\theta}^{2} / r^{2}-\left(D U_{\theta}\right)^{2}-\left(D U_{z}\right)^{2}\right)}{\left(r D U_{\theta}-U_{\theta}\right)^{2}+r^{2}\left(D U_{z}\right)^{2}}\right|_{r_{0}} .
$$

This function reaches a maximum $\sigma_{\max }$ at $r_{0}=r_{c}$, thereby corresponding to a helix angle $\beta$ of the wave vector given by (38) evaluated at $r_{c}$.

As can be seen from expression (38), these generalized centrifugal modes are not captured by models with discontinuous velocity profiles since they require, among other things, $D U_{z} \equiv d U_{z} / d r$ to be nonzero at a particular location $r_{c}$. Although the present model captures well the $\mathrm{K}-\mathrm{H}$ mode and the physical effect of a finite shear layer can be accounted for by heuristic arguments, it, however, totally misses the generalized centrifugal modes that destabilize large negative spiral modes with $\beta=-k / m=\cos t$ when $m$ $\rightarrow \infty$.

It is interesting to put our temporal stability results in perspective with the spatial ones of Lu and Lele. ${ }^{43}$ These authors have studied a smooth profile that consists of a jet with strong coflow associated to an annular swirling flow. The presence of the coflow renders the flow hyperconvective and spatial and temporal stability results become very similar. They can be deduced easily one from each other through the Gaster transformation. ${ }^{44} \mathrm{Lu}$ and Lele ${ }^{43,45}$ have found that the unstable modes on these profiles separate into two families: the so-called $\mathrm{K}-\mathrm{H}$ modes due to shear instability and the centrifugal modes. They have also shown that both type of instabilities can reach growth rates with the same order of magnitude. Our results obtained on a discontinuous model vortex could serve as a guideline to understand the mode selection among the $\mathrm{K}-\mathrm{H}$ modes with different $m$, if one keeps in mind the modifications brought by nonzero shear layer thicknesses. The present model, however, fails to give any insight on the dynamics of generalized centrifugal modes described by the asymptotic theory of Leibovich and Stewartson. ${ }^{17}$

From these physical considerations we conclude that some caution should be taken with the spatio-temporal analysis of the discontinuous model. When absolute transition occurs for a finite $m$ and finite $k$ we may reasonably hope that a similar transition will occur for realistic continuous profiles but when transition occurs through $m \rightarrow \infty$ as for the jet at high swirl, we know that the results will not be robust since the introduction of finite shear will stabilize this mode and destabilize through general centrifugal instability azimuthal modes with large negative wave number $m$ that are completely absent in the present model.

\section{ACKNOWLEDGMENTS}

The authors wish to thank T. Loiseleux, P. Huerre, and P. Schmid for stimulating discussions.

\section{APPENDIX A: ASYMPTOTIC STABILITY ANALYSIS FOR $m=0$ and $k \ll 1$}

Our goal in this appendix is to derive the asymptotic expansion of the bulging mode $m=0$ at $k \ll 1$. Assuming $\beta$ of order unity, we get $\omega_{j}^{2} \sim 4 S^{2} k^{2} / \beta^{2}$. Using the Bessel function expansion,

$$
\lim _{k \rightarrow 0} k \frac{K_{0}^{\prime}(k)}{K_{0}(k)}=\frac{1}{\ln (k)}+O(1),
$$

the dispersion relation, Eq. (21), becomes

$$
\frac{J_{0}^{\prime}(\beta)}{J_{0}(\beta)}-\frac{4}{\left(1-\Delta^{2}\right)(\beta)}=F(\beta)=0
$$

Plotting of the isocontours of $\operatorname{Re}[F(\beta)]$ and $\operatorname{Im}[F(\beta)]$ in the complex plane, one verifies that the equation $F(\beta)=0$ has 


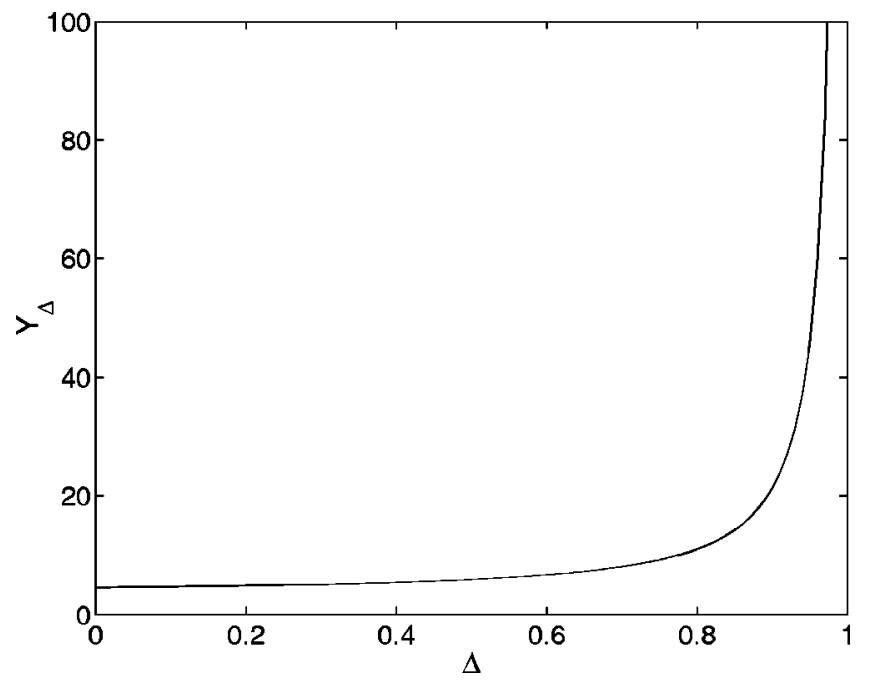

FIG. 15. Numerical determination of $Y_{\Delta}$ (see the text).

only solutions for $\beta$ real (Kelvin waves) and $\beta$ purely imaginary. The equation has only one solution of positive imaginary part corresponding to an unstable mode for $m=0, \beta$ $=i Y_{\Delta}$,

$$
\omega \sim(1+a) k+i \frac{2 S}{Y_{\Delta}} k .
$$

Equation (A2) is solved numerically for $\Delta \in[0 ; 1]$ and results are displayed in Fig. 15. We note that, since the intensity of the instability is inversely proportional to $Y_{\Delta}$, it increases as the jump in azimuthal velocity $(1-\Delta)$ increases. As $\Delta$ approaches one, $Y_{\Delta}$ tends to infinity and the growth rate is "weaker" than linear in $k$, as shown by LCH.

\section{APPENDIX B: ASYMPTOTIC STABILITY ANALYSIS FOR $m= \pm 1$ and $k \ll 1$}

In this appendix, we calculate the asymptotic expansion of the bending modes $m= \pm 1$ for $k \ll 1$. Using the expansion of Bessel functions,

$$
\lim _{k \rightarrow 0} k \frac{K_{ \pm 1}^{\prime}(k)}{K_{ \pm 1}(k)}=-1-k^{2}\left(K-\frac{1}{4}\right)+O\left(k^{4}\right),
$$

where $K=\log (2 / k)-C+1 / 4$ and $C=0.5772$ is the Euler constant, we first develop the dispersion relation (21) to cubic order in $k$ :

$$
\begin{aligned}
\omega_{j}^{3}(2 & \left.+k^{2} K\right)+\omega_{j}^{2}\left(4 S+2 k+\frac{(1+4 K) S}{2} k^{2}+\frac{k^{3}}{2}+o\left(k^{4}\right)\right) \\
& +\omega_{j}\left(2 S^{2}+2 S k+\left(1+K S^{2}+\frac{5 S^{2}}{4}\right) k^{2}+\frac{3 S}{2} k^{3}+o\left(k^{4}\right)\right) \\
& +S^{3} k^{2}+S^{2} k^{3}+o\left(k^{4}\right)=0 .
\end{aligned}
$$

The stability of the mode is determined by the existence of complex roots of this polynomial of order 3 . We encounter stability if there exist only real roots and have instability otherwise. A generalized "discriminant" $p$ of third-order polynomials can be defined, whose sign determines the na- ture of the roots. If $p>0$, there are two complex-conjugate solutions, if $p<0$, all roots are real. For expression (B2), $p$ equals to third order in $k$,

$$
p=\frac{S^{4}\left(2-S^{2}\right)}{216} k^{2}+\frac{m S^{3}\left(S^{2}-4\right)}{216} k^{3}+0\left(k^{4}\right) .
$$

One can easily verify that at small $k, p(k)$ is positive for $S<\sqrt{2}$ and negative for $S>\sqrt{2}$, independently of the sign of $m= \pm 1$. When the bending modes are unstable at small $k$, i.e., when $S<\sqrt{2}$, their frequency and growth rate is given by

$$
\begin{aligned}
\omega_{ \pm 1} \sim\left(a+\frac{1}{2}\right) k+\alpha \frac{S}{8} k^{2} & \\
& +i\left(\frac{\sqrt{1-\frac{S^{2}}{2}}}{2} k+\alpha \frac{S}{8 \sqrt{1-\frac{S^{2}}{2}}} k^{2}\right) .
\end{aligned}
$$

${ }^{1} \mathrm{~J}$. McWilliams, "The emergence of isolated vortices in turbulent flow," J. Fluid Mech. 146, 21 (1984).

${ }^{2}$ A. Roshko, "Structure of turbulent shear flows: a new look," AIAA J. 14, 1349 (1976).

${ }^{3}$ M. McIntyre, "On the Antartic ozone hole," J. Atmos. Terr. Phys. 51, 19 (1989).

${ }^{4} \mathrm{X}$. Carton and J. McWilliams, "Barotropic and baroclinic instabilities of axisymmetric vortices in a quasi-geostrophic model," in Mesoscale/ Synoptic Coherent Structures in Geophysical Turbulence, edited by J. Nihoul and B. Jamart (Elsevier, Amsterdam, 1989), pp. 225-244

${ }^{5} \mathrm{G}$. Carnevale and R. Kloosterziel, "Emergence and evolution of triangular vortices," J. Fluid Mech. 259, 305 (1994).

${ }^{6}$ K. Bergeron, E. Coutsias, J. Lynov, and A. Nielsen, "Self-organization in circular shear layers," Phys. Scr. T67, 33 (1996).

${ }^{7}$ M. Rabaud and Y. Couder, "A shear-flow instability in circular geometry," J. Fluid Mech. 136, 291 (1983).

${ }^{8}$ J.-M. Chomaz, M. Rabaud, C. Basdevant, and Y. Couder, "Experimental and numerical investigation of a forced circular shear layer," J. Fluid Mech. 187, 115 (1988).

${ }^{9}$ W.-G. Früh and P. Read, "Experiments on a barotropic rotating shear layer. Part 1. Instability and steady vortices," J. Fluid Mech. 383, 143 (1999).

${ }^{10}$ J. van de Konijnenberg, A. Nielsen, J. Rasmussen, and B. Stenum, "Shear flow instability in a rotating fluid," J. Fluid Mech. 387, 177 (1999).

${ }^{11}$ R. Kloosterziel and G. van Heijst, "An experimental study of unstable barotropic vortices in a rotating fluid," J. Fluid Mech. 223, 1 (1991).

${ }^{12}$ P. Marcus, "Vortex dynamics in a shearing zonal flow," J. Fluid Mech. 215, 393 (1990).

${ }^{13}$ Y. Afanasyev and W. Peltier, "Three-dimensional instability of swirling flow in rotating fluid: Laboratory experiments and related theoretical predictions," Phys. Fluids 10, 3194 (1998)

${ }^{14}$ A. Colette, P. Billant, B. Beaudoin, J. Boulay, S. Lassus-Pigat, C. Niclot, H. Schaffner, and J.-M. Chomaz, "Instabilities of a vertical columnar vortex pair in a strongly stratified and rotating fluid," 53rd Meeting of the APS Division of Fluid Dynamics, Washington DC, November 2000, pp. $19-21$.

${ }^{15}$ P. Drazin and W. Reid, Hydrodynamic Stability (Cambridge University Press, Cambridge, 1981).

${ }^{16}$ H. Ludwieg, "Stabilität der Strömung in einem zylindrischen Ringraum," Z. Flugwiss. 8, 135 (1960).

${ }^{17}$ S. Leibovich and K. Stewartson, "A sufficient condition for the instability of columnar vortices," J. Fluid Mech. 126, 335 (1983).

${ }^{18}$ J. T. Snow, "On inertial instability as related to the multiple vortex phenomenon," J. Atmos. Sci. 35, 1660 (1978).

${ }^{19}$ P. Billant, J.-M. Chomaz, and P. Huerre, "Experimental study of vortex breakdown in swirling jets," J. Fluid Mech. 376, 183 (1998).

${ }^{20}$ M. Escudier, J. Bornstein, and T. Maxworthy, "The dynamics of confined vortices," Proc. R. Soc. London, Ser. A 382, 335 (1982). 
${ }^{21}$ M. Lessen, P. J. Singh, and F. Paillet, "The stability of a trailing line vortex. Part 1. Inviscid theory," J. Fluid Mech. 63, 753 (1974).

${ }^{22} \mathrm{E}$. Mayer and K. Powell, "Viscous and inviscid instabilities of a trailing line vortex," J. Fluid Mech. 245, 91 (1992).

${ }^{23}$ M. R. Khorrami, "On the viscous modes of instability of a trailing line vortex," J. Fluid Mech. 225, 197 (1991).

${ }^{24}$ C. Olendraru, A. Sellier, M. Rossi, and P. Huerre, "Absolute/convective instability of the Batchelor vortex," C. R. Acad. Sci., Ser. IIb 323, 153 (1996).

${ }^{25}$ C. Olendraru, A. Sellier, and P. Huerre, "Inviscid instability of the Batchelor vortex: Absolute/convective transition and spatial branches," Phys. Fluids 11, 1805 (1999).

${ }^{26}$ I. Delbende, J.-M. Chomaz, and P. Huerre, "Absolute/convective instabilities in the Batchelor vortex: a numerical study of the linear impulse response," J. Fluid Mech. 355, 229 (1998).

${ }^{27}$ X. Yin, D. Sun, M. Wei, and J. Wu, "Absolute and convective instability character of slender viscous vortices," Phys. Fluids 12, 1062 (2000).

${ }^{28}$ T. Loiseleux, J.-M. Chomaz, and P. Huerre, "The effect of swirl on jets and wakes: Linear instability of the Rankine vortex with axial flow," Phys. Fluids 10, 1120 (1998).

${ }^{29}$ J. E. Martin and E. Meiburg, "On the stability of the swirling jet shear layer," Phys. Fluids 6, 424 (1994).

${ }^{30}$ T. Loiseleux, I. Delbende, and P. Huerre, "Absolute and convective instabilities of a swirling jet/wake shear layer," Phys. Fluids 12, 375 (2000).

${ }^{31} \mathrm{D}$. Lim and L. Redekopp, "Absolute instability conditions for variable density, swirling jet flows," Eur. J. Mech. B/Fluids 17, 165 (1998).

${ }^{32}$ P. Monkewitz and K. Sohn, "Absolute instability in hot jets," AIAA J. 26, 911 (1988)

${ }^{33}$ C. M. Ho and P. Huerre, "Perturbed shear layers," Annu. Rev. Fluid Mech. 16, 365 (1984).
${ }^{34}$ P. G. Saffman, Vortex Dynamics (Cambridge University Press, Cambridge, UK, 1992).

${ }^{35}$ L. Kelvin, "Vibrations of a columnar vortex," Philos. Mag. 10, 155 (1880) (Kelvin's name was W. Thomson).

${ }^{36}$ P. Huerre and P. A. Monkewitz, "Local and global instabilities in spatially developing flows," Annu. Rev. Fluid Mech. 22, 473 (1990).

${ }^{37}$ R. J. Briggs, Electron-Stream Interaction with Plasmas (MIT Press, Cambridge, MA, 1964).

${ }^{38} \mathrm{P}$. Huerre and P. A. Monkewitz, "Absolute and convective instabilities in free shear layers," J. Fluid Mech. 159, 151 (1985).

${ }^{39}$ H. B. Squire, "Analysis of the vortex breakdown phenomenon," Part I, Aerospace Department, Imperial College, Report No. 102 (1960).

${ }^{40} \mathrm{~T}$. B. Benjamin, "Theory of the vortex breakdown phenomenon," J. Fluid Mech. 14, 593 (1962)

${ }^{41} \mathrm{P}$. Monkewitz, "The absolute and convective nature of instability in twodimensional wakes at low Reynolds numbers," Phys. Fluids 31, 999 (1988).

${ }^{42}$ T. F. Balsa, "On the spatial instability of piecewise linear free shear layers,” J. Fluid Mech. 174, 553 (1987).

${ }^{43} \mathrm{G}$. Lu and S. Lele, "Inviscid instability of compressible swirling mixing layers," Phys. Fluids 11, 450 (1999).

${ }^{44} \mathrm{M}$. Gaster, "A note on the relation between temporally-increasing and spatially-increasing disturbances in hydrodynamic stability," J. Fluid Mech. 14, 222 (1962).

${ }^{45}$ This change in sign of $d \sigma / d k$ guarantees continuity of the growth rate, if we break with convention and use a signed $k$ and only positive $m$, as done by Lu and Lele (Ref. 43). 